\title{
Remnant mass, spin, and recoil from spin aligned black-hole binaries
}

\author{
James Healy, Carlos O. Lousto, and Yosef Zlochower \\ Center for Computational Relativity and Gravitation, \\ School of Mathematical Sciences, Rochester Institute of Technology, \\ 78 Lomb Memorial Drive, Rochester, New York 14623
}

(Dated: July 1, 2014)

\begin{abstract}
We perform a set of 36 nonprecessing black-hole binary simulations with spins either aligned or counteraligned with the orbital angular momentum in order to model the final mass, spin, and recoil of the merged black hole as a function of the individual black hole spin magnitudes and the mass ratio of the progenitors. We find that the maximum recoil for these configurations is $V_{\max }=526 \pm 23 \mathrm{~km} \mathrm{~s}^{-1}$, which occurs when the progenitor spins are maximal, the mass ratio is $q_{\max }=m_{1} / m_{2}=0.623 \pm 0.038$, the smaller black-hole spin is aligned with the orbital angular momentum, and the larger black-hole spin is counteraligned $\left(\alpha_{1}=-\alpha_{2}=1\right)$. This maximum recoil is about $80 \mathrm{~km} \mathrm{~s}^{-1}$ larger than previous estimates, but most importantly, because the maximum occurs for smaller mass ratios, the probability for a merging binary to recoil faster than $400 \mathrm{~km} \mathrm{~s}^{-1}$ can be as large as $17 \%$, while the probability for recoils faster than $250 \mathrm{~km} \mathrm{~s}^{-1}$ can be as large as $45 \%$. We provide explicit phenomenological formulas for the final mass, spin, and recoil as a function of the individual BH spins and the mass difference between the two black holes. Here we include terms up through fourth-order in the initial spins and mass difference, and find excellent agreement (within a few percent) with independent results available in the literature. The maximum radiated energy is $E_{\mathrm{rad}} / m \approx 11.3 \%$ and final spin $\alpha_{\mathrm{rem}}^{\max } \approx 0.952$ for equal mass, aligned maximally spinning binaries.
\end{abstract}

PACS numbers: 04.25.dg, 04.25.Nx, 04.30.Db, 04.70.Bw

\section{INTRODUCTION}

The fully nonlinear simulations of merging black-hole binaries (BHBs) that were enabled by the 2005 breakthroughs in numerical relativity [1-3] revealed some unexpectedly large effects. Perhaps one of the most striking is that the merger remnant can recoil away from the center of mass by thousands of $\mathrm{km} / \mathrm{s}$ for BHBs with spins at least partially in the orbital plane [4 8 . Such recoils, if common, would have major implications for structure formation and the evolution of galaxies, as well as the retention of BHs in globular clusters and the formation of intermediate mass BHs. The probability of these large recoils depends on the distribution of mass ratios and spins of the progenitor binaries. While the detailed modeling of those recoil velocities from merging BHBs as a function of the individual spins (magnitudes and directions) of the BHs and the mass ratio is well underway [8] 12 , the major effort required to simulate BHBs in a realistic astrophysical environment started more recently [13 24]. Analyses of Newtonian and post-Newtonian simulations appear to indicate that accretion dynamics will skew the spin distributions away from configurations that favor very large recoils [25 27] because these effects tend to align (or counter-align) the spins with the orbital angular momentum. In addition, during the late stages of the BHB evolution, post-Newtonian resonance effects 22, 29. tend to further align the $\mathrm{BH}$ spins with each other and the orbital angular momentum (or counteralign them azimuthally). On the other hand, recent studies of chaotic [30] and partially chaotic 31] accretion suggest misalignment of spins can also be a common evolutionary scenario for BHBs, possibly allowing for the merger remnant to escape from large galaxies $[32,33$.

In this paper we simulate the late-inspiral and merger stages of BHBs in configurations where the spins are exactly aligned or counter-aligned with the orbital angular momentum. By doing so, we are able to quantify how large the recoil can be when coherent accretion effects dominate the distribution of spin directions, thus providing a lower bound to the recoil of the $\mathrm{BH}$ remnant. The aligned-spin case also provides the optimal configuration for the radiation of gravitational energy and angular momentum. Here we provide a unified, higher-order phenomenological model of the remnant mass, spin, and recoil from the merger of two BHs with different masses and different spin magnitudes (either aligned or antialigned spins).

This paper is organized as follows, in Sec. II we review the current status of the modeling of the remnant recoil. In Sec. III we review the numerical techniques used for our evolutions of the BHBs and the subsequent analyses of the progenitor and remnant properties. In Sec. IV] we present the explicit form of the new phenomenological formulas for the final mass, spin and recoil of the merger remnant. We apply these formulas to astrophysically motivated distributions of the mass ratios and spins of the progenitor binaries to obtain probabilities for a given recoil, final remnant mass and spin. In Sec. VI we discuss the consequences of our results. 


\section{MODEL OF RECOILS ON THE ORBITAL PLANE}

Beginning in Ref. 4, we developed a heuristic model for the gravitational recoil of a merging binary. The model for the in-plane recoil was based on PN-inspired fitting formulas combined with the results of 34-36 (a similar model was developed independently in [37]). Here we use the PN-inspired variables

$$
\begin{array}{r}
m=m_{1}+m_{2}, \\
\delta m=\frac{m_{1}-m_{2}}{m}, \\
\vec{S}=\vec{S}_{1}+\vec{S}_{2}, \\
\vec{\Delta}=m\left(\vec{S}_{2} / m_{2}-\vec{S}_{1} / m_{1}\right),
\end{array}
$$

where $m_{i}$ is the mass of BH $i=1,2$ and $\vec{S}_{i}$ is the spin of $\mathrm{BH} i$. We also use the auxiliary variables

$$
\begin{array}{r}
\eta=\frac{m_{1} m_{2}}{m^{2}} \\
q=\frac{m_{1}}{m_{2}} \\
\vec{\alpha}_{i}=\vec{S}_{i} / m_{i}^{2}
\end{array}
$$

where $\left|\vec{\alpha}_{i}\right| \leq 1$ is the dimensionless spin of $\mathrm{BH} i$, and we use the convention that $m_{1} \leq m_{2}$ and hence $q \leq 1$.

The in-plane recoil can be split (at least approximately) into two components: a part due solely to unequal masses and a part due to the out-of-plane components of the spins of the two BHs. To lowest order in the spin, the formula is given by,

$$
\vec{V}_{\text {recoil }}\left(q, \vec{\alpha}_{i}\right)=v_{m} \hat{e}_{1}+v_{\perp}\left(\cos (\xi) \hat{e}_{1}+\sin (\xi) \hat{e}_{2}\right)
$$

where

$$
\begin{gathered}
v_{m}=-A \eta^{2} \delta m(1+B \eta), \\
v_{\perp}=H \eta^{2}\left[\frac{\Delta_{\|}}{m^{2}}-H_{S} \delta m \frac{S_{\|}}{m^{2}}\right] .
\end{gathered}
$$

Here the index $\perp$ and $\|$ refer to components perpendicular to and parallel to the orbital angular momentum during the short period around merger when most of the recoil is generated, while $\hat{e}_{1}, \hat{e}_{2}$ are orthogonal unit vectors in the orbital plane, and $\xi$ measures the angle between the "unequal mass" and "spin" contributions to the recoil velocity in the orbital plane (See Fig. 1). This formula can be extended by adding additional nonlinear terms (as we will show in this paper). The coefficients are given by $A=1.2 \times 10^{4} \mathrm{~km} \mathrm{~s}^{-1}[34, B=-0.93$ [34], and $H=(6.9 \pm 0.5) \times 10^{3} \mathrm{~km} \mathrm{~s}^{-1} 38$. We will study in detail how $\xi$ depends on the configurations here $(\xi$ was initially studied in Ref. 38, where it was found that $\xi \sim 145^{\circ}$ for a range of quasicircular configurations).

\section{A. Post-Newtonian analysis}

Here we use the leading-order post-Newtonian expressions for the radiated linear momentum to get a qualitative understanding of the full numerical results. As seen in Eq. (3.31) of Ref. 39, the instantaneous radiated linear momentum due to the asymmetry in the masses of the binary is given by

$$
\begin{aligned}
\dot{\vec{P}}_{N}= & -\frac{8 \eta^{2} \delta m}{105}\left(\frac{m}{r}\right)^{4}\left[\left(5 V_{T}^{2}-2 V_{r}^{2}+4 m / r\right) V_{r} \hat{n}\right. \\
& \left.-\left(12 V_{r}^{2}+50 V_{T}^{2}+8 m / r\right) V_{T} \hat{\lambda}\right]
\end{aligned}
$$

and the radiated linear momentum due to the leadingorder spin-orbit coupling is

$$
\dot{\vec{P}}_{S O}=-\frac{8 \eta^{2} m}{15 r^{5}}\left[4 V_{T} V_{r} \hat{n}+2\left(V_{T}^{2}-V_{r}^{2}\right) \hat{\lambda}\right] \Delta_{\|},
$$

where $V_{T}$ and $V_{r}$ are the tangential and radial velocities, respectively. The velocity is given by, $\vec{V}=V_{T} \hat{\lambda}+V_{r} \hat{n}$, where $\hat{n}=\left(\vec{r}_{1}-\vec{r}_{2}\right) /\left|\vec{r}_{1}-\vec{r}_{2}\right|$ and $\hat{n} \times \hat{\lambda}=\hat{L}$.

For a quasicircular orbit, the angle between these two components of the instantaneous radiated linear momentum is given by

$$
\cos \xi^{\Delta}=\left[-1+\frac{15625}{6728} \frac{V_{r}^{2}}{V_{T}^{2}}+\ldots\right] \operatorname{sign}(\delta m \vec{\Delta} \cdot \hat{L}),
$$

and hence, for circular orbits, i.e. $V_{r}=0$,

$$
\cos \xi_{c}^{\Delta}=-\operatorname{sign}(\delta m \vec{\Delta} \cdot \hat{L})
$$

Thus the two components are opposite of each other when $\vec{\Delta}$ is aligned with the orbital angular momentum (corotating orbits for our configurations). Similarly, for our counter-rotating configurations, the two components add constructively.

On the other hand, for orbits dominated by the radial motion instead (i.e., $V_{T} \ll V_{r}$ ) the angle $\xi$ has the form,

$$
\cos \xi^{\Delta}=\left[\frac{4 V_{T}}{V_{r}} \frac{\left(r V_{r}^{2}+2 M\right)}{\left(-r V_{r}^{2}+2 M\right)}+\ldots\right] \operatorname{sign}(\delta m \vec{\Delta} \cdot \hat{L})
$$

Hence, in the near-headon case (i.e. $V_{T} \approx 0$ ), we have

$$
\cos \xi_{h}^{\Delta} \approx 0
$$

and the two components of the recoil are perpendicular to each other.

The next leading term in the spin orbit contribution to the recoil [see Eqs. (4.7)-(4.9) of Ref. 40]] is proportional to $\delta m S_{\|}$. For circular orbits the angle $\cos \xi_{c}^{S}$ between the unequal mass recoil and the terms in the recoil proportional to $\delta m \vec{S}$ is given by

$$
\cos \xi_{c}^{S}=-\operatorname{sign}(\vec{S} \cdot \hat{L}),
$$

while for headon collisions [see Eqs. (3.17) of Ref. [40] the two components are perpendicular and

$$
\cos \xi_{h}^{S} \approx 0
$$




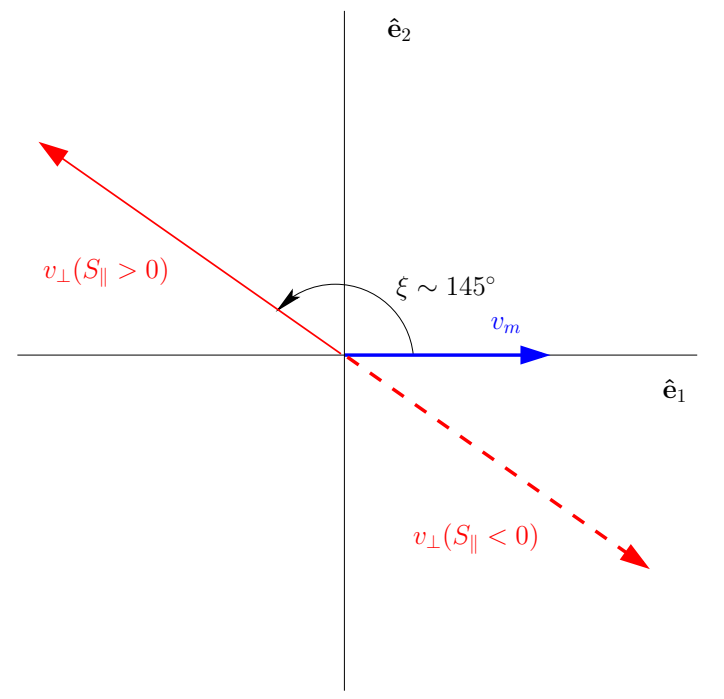

FIG. 1: A sketch showing how the angle $\xi$ between the unequal mass contribution to the recoil and the spin dependent contribution to the recoil depends on the sign of $S_{\|}$(with similar behavior for the $\delta m \Delta_{\|}$dependent term). The two components essentially add if the net spin is counteraligned with the orbital angular momentum and subtract if the spin is aligned with the orbital angular momentum.

In a full numerical simulation, the inspiral of two BHs is neither circular nor headon, and hence we expect a value of $\xi$ that lies between $90^{\circ}$ and either $180^{\circ}$ (corotating) or $0^{\circ}$ (counterrotating) (see Fig. 1). In particular, because of the hangup effect [41] we expect that alignedspin configurations, which have tighter (i.e., more circular) orbits, have $\xi \approx 180^{\circ}$, while counter-aligned configurations, which inspiral much more quickly, should have $\xi \lesssim 90^{\circ}$.

Based on the above analysis, we expect $\cos \xi$ to be a discontinuous function with a finite jump when $\vec{S} \cdot \vec{L}$ and $\delta m \Delta \cdot \vec{L}$ change sign. While we can model $\xi$ as a discontinuous function, there is a way around this. Note that the magnitude of the in-plane recoil is given by

$$
V^{2}=\left(v_{m}+\left|v_{\perp}\right| \cos \xi\right)^{2}+v_{\perp}^{2} \sin ^{2} \xi
$$

where $\left|v_{\perp}\right|$ is the magnitude of spin contribution to the in-plane recoil. The important thing to note is that while $\cos \xi$ is discontinuous, the recoil itself should be continuous. For this to be true, the sign change in $\cos \xi$ can only occur when $v_{\perp}=0$, i.e., we expect that $\left|v_{\perp}\right| \cos \xi$ is continuous. We can therefore express the product $\left|v_{\perp}\right| \cos \xi$ as a product of two continuous functions $v_{\perp}$, which we will allow to be positive or negative, and $\cos \tilde{\xi}$, where $\cos \tilde{\xi}$ has a fixed sign (for historical reasons, we chose $\cos \tilde{\xi}$ to be negative) and $|\cos \tilde{\xi}|=|\cos \xi|$. Finally, the magnitude of the recoil is given by,

$$
V^{2}=\left(v_{m}+v_{\perp} \cos \tilde{\xi}\right)^{2}+v_{\perp}^{2} \sin ^{2} \tilde{\xi},
$$

where $v_{\perp}$ can be negative and $\operatorname{sign}\left(v_{\perp}\right) \cos \tilde{\xi}$ is the cosine of the angle between unequal-mass and spin components of the recoil.

\section{NUMERICAL SIMULATIONS}

We use the TwoPunctures thorn [42] to generate initial puncture data 43 for the BHB simulations. These data are characterized by mass parameters $m_{p}$ (which are not the horizon masses), as well as the momentum and spin, of each BH. We evolve these BHB data sets using the LAzEv 44 implementation of the moving puncture approach [2, 3] with the conformal function $W=\sqrt{\chi}=$ $\exp (-2 \phi)$ suggested by Ref. 45. For the runs presented here, we use centered, eighth-order finite differencing in space [46] and a fourth-order Runge Kutta time integrator. (Note that we do not upwind the advection terms.) Our code uses the Cactus/EinsteinToolkit [47, 48] infrastructure. We use the CARPET [49] mesh refinement driver to provide a "moving boxes" style of mesh refinement.

We locate the apparent horizons using the AHFInDERDIRECT code [50] and measure the horizon spin using the isolated horizon (IH) algorithm detailed in [51].

For the computation of the radiated energy and linear momentum we use the formulas in 52] which are expressed directly in terms of the Weyl scalar $\psi_{4}$. To extract the radiation of angular momentum components, we use formulas based on "flux-linkages" 53 , and explicitly written in terms of $\psi_{4}$ in [52, 54].

To generate the initial data parameters, we use 3PN quasicircular orbital parameters with a given initial orbital frequency $\omega_{i}$. In practice this leads to an initial eccentricity of the order of $e_{i} \sim 10^{-2}$ that radiates after a few orbits to about $e_{f} \sim 10^{-3}$, which is small enough for modeling the remnant in astrophysical applications. Table III provides explicit values for all the initial data parameters used in each of the runs presented in this paper. We also provide the initial and final eccentricity and total number of orbits in the table.

We evolve these data sets using the grid refinement structure and global resolution discussed in the Appendix. In the Appendix, we also describe in detail the errors in our results due to finite extraction radii and finite truncation errors, as well as how we extrapolate from finite radii to null infinity.

In order to cover the three-dimensional parameter space of the aligned-spin BHBs, we consider several families of physically motivated configurations. We denote our configurations by $\mathrm{XY}$, where $\mathrm{X}=\mathrm{U}, \mathrm{D}$, or 0 denotes the spin of the smaller BH (i.e., aligned, counteraligned, or zero) and $\mathrm{Y}$ denotes the spin of the larger $\mathrm{BH}$. If accretion tends to align the spins, then the UD, DU, UU, and DD configurations should be among the most probable. The $0 \mathrm{U}$ and $0 \mathrm{D}$ configurations, depicted in Fig. 2, are interesting in that if the recoil as given in Eq. (2b) is dominated by the leading $\Delta_{\|}$dependence, then a $0 \mathrm{U}$ or $0 \mathrm{D}$ configuration is an effective counterpart to a UU 


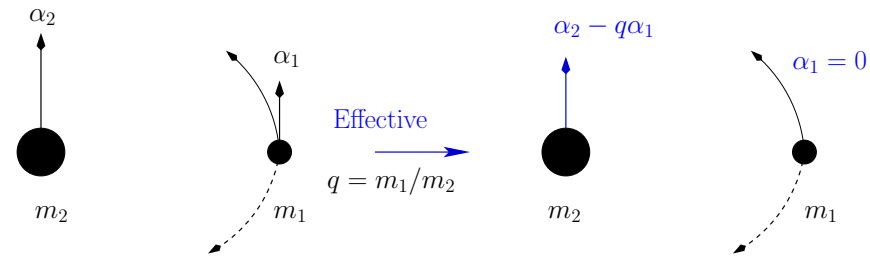

FIG. 2: The (counter)aligned spin configuration UU (DD) and its effective counterpart 0U (0D) (dashed counterorbiting).

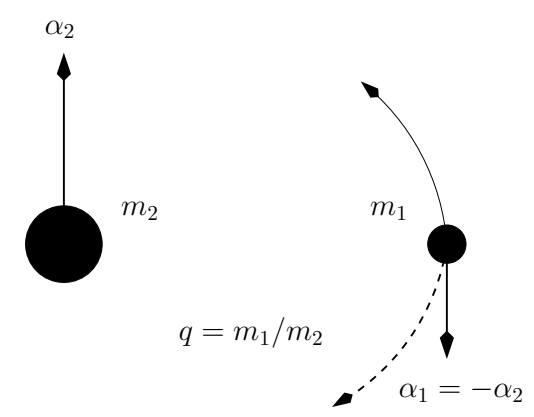

FIG. 3: The DU and UD (dashed counterorbiting) configurations.

or DD configuration where both $\mathrm{BHs}$ are spinning with the same dimensionless spin $\alpha$ and $\alpha_{\text {Effective }}= \pm(1-q) \alpha^{\prime}$ (i.e., a $0 \mathrm{U}$ with spin $\alpha_{\text {Effective }}$ should give the same recoil

Since the initial data assumes conformal flatness and pure longitudinal extrinsic curvature, it contains initial distortions that are either radiated away or absorbed by the BHs during the first orbital period. After this initial transient period, the $\mathrm{BH}$ masses and spins settle to their equilibrium values. In Table II] we give the values for the horizon mass and spin after this transient period has ended.

After the BHs in the progenitor BHB merge, we measure the remnants mass, spin, and recoil velocity. We measure the recoil velocity from the radiation of linear momentum at infinity, as this is the most reliable and gauge invariant way of computing recoils. The resulting recoil velocities are given in Table IV. In order to produce

In Table $\mathrm{V}$, we give the horizon mass and spin magnitude of the remnant $\mathrm{BH}$ for each configuration studied here. We measure these using both the isolated horizon formalism and based on the measured radiated mass and angular momentum. However, the isolated horizon measurements are expected to be more accurate, and the differences between the isolated horizon and radiation quantities is largely due to truncation errors in the radiation as a UU/DD configuration with $\left.\alpha_{2}=\alpha_{1}=\alpha^{\prime}\right)$. Since $\alpha_{\text {Effective }}$ is smaller than $\alpha^{\prime}$ we can apparently simulate maximal UU and DD configurations with non-maximal $0 \mathrm{U}$ and $0 \mathrm{D}$ configurations. We thus study BHBs with the smaller $\mathrm{BH}$ nonspinning and the larger $\mathrm{BH}$ spinning with spin $\alpha_{\text {Effective }}= \pm(1-q)$ for $q=1 / 2,1 / 3,1 / 4,1 / 5,1 / 6$. A first analysis of those simulations suggested that although leading, the $\Delta_{\|}$dependence in Eq. $2 \mathrm{~b}$ is not sufficient to model the recoils with high accuracy. We thus consider additional families (see Fig. 3 ) of BHBs, with specific spins $\alpha \leq 0.8$ in a UD or DU configuration. In addition to showing the importance of the total spin $S_{\|}$to the recoil, we also found from these configurations that the maximum recoil occurs for $q_{\max } \sim 0.62$, as shown in Fig. 4 .

We chose other configurations to selectively activate or deactivate blocks of terms in the expansion formulas for the recoil and radiated energy-momentum. Thus some simulations have only one of the variables $\delta m, S_{\|}$, and $\Delta_{\|}$nonvanishing and others have all of them nonvanishing. This provides a means of fitting all terms and then verifying the fit for more general cases. The complete set of initial data parameters are given in Table I. In the table, the runs are labeled by the mass ratio, spin magnitude of the black hole 1 (the smaller $\mathrm{BH}$ ) and spin magnitude of the spin 2 (the larger $\mathrm{BH}$ ).

accurate results, we extracted the waveform at different finite radii and extrapolated to infinity. Here we chose observer locations equidistant in $1 / r$, where the largest extraction radius was $102.6 \mathrm{~m}$. We fit the finite-radius results for the recoil, energy radiated, and angular momentum radiated as a linear and quadratic function in $1 / r$, and we use the difference between these two fits as an estimate for the error. As we discuss in the Appendix, other sources of error come from the finite numerical resolution and the maximum $\ell$-mode used in the extraction. Based on our assessment of those errors (see Appendix), we compute the recoil using the $\ell=2$ through $\ell=6$ modes.

zone that do not affect the accuracy near the horizons themselves (see Ref. [55]). 
TABLE I: Initial data parameters for the quasi-circular configurations with a non-spinning smaller mass black hole (labeled 1 ), and a larger mass spinning black hole (labeled 2). The punctures are located at $\vec{r}_{1}=\left(x_{1}, 0,0\right)$ and $\vec{r}_{2}=\left(x_{2}, 0,0\right)$, with momenta $P= \pm(0, P, 0)$, spins $\vec{S}_{i}=\left(0,0, S_{i}\right)$, mass parameters $m^{p} / m$, horizon (Christodoulou) masses $m^{H} / m$, total ADM mass $M_{\mathrm{ADM}}$, and dimensionless spins $a / m_{H}=S / m_{H}^{2}$. The configuration are denoted by QX_Y_Z, where X gives the mass ratio $m_{1}^{H} / m_{2}^{H}$, Y gives the spin of the smaller $\mathrm{BH}\left(a_{1} / m_{H}^{2}\right)$, and $\mathrm{Z}$ gives the spin of the larger $\mathrm{BH}\left(a_{2} / m_{H}^{2}\right)$. $(*)$ Note that the $q=1 / 10$ binary also had an initial radial momentum of $P_{r} / m=-0.0001685$.

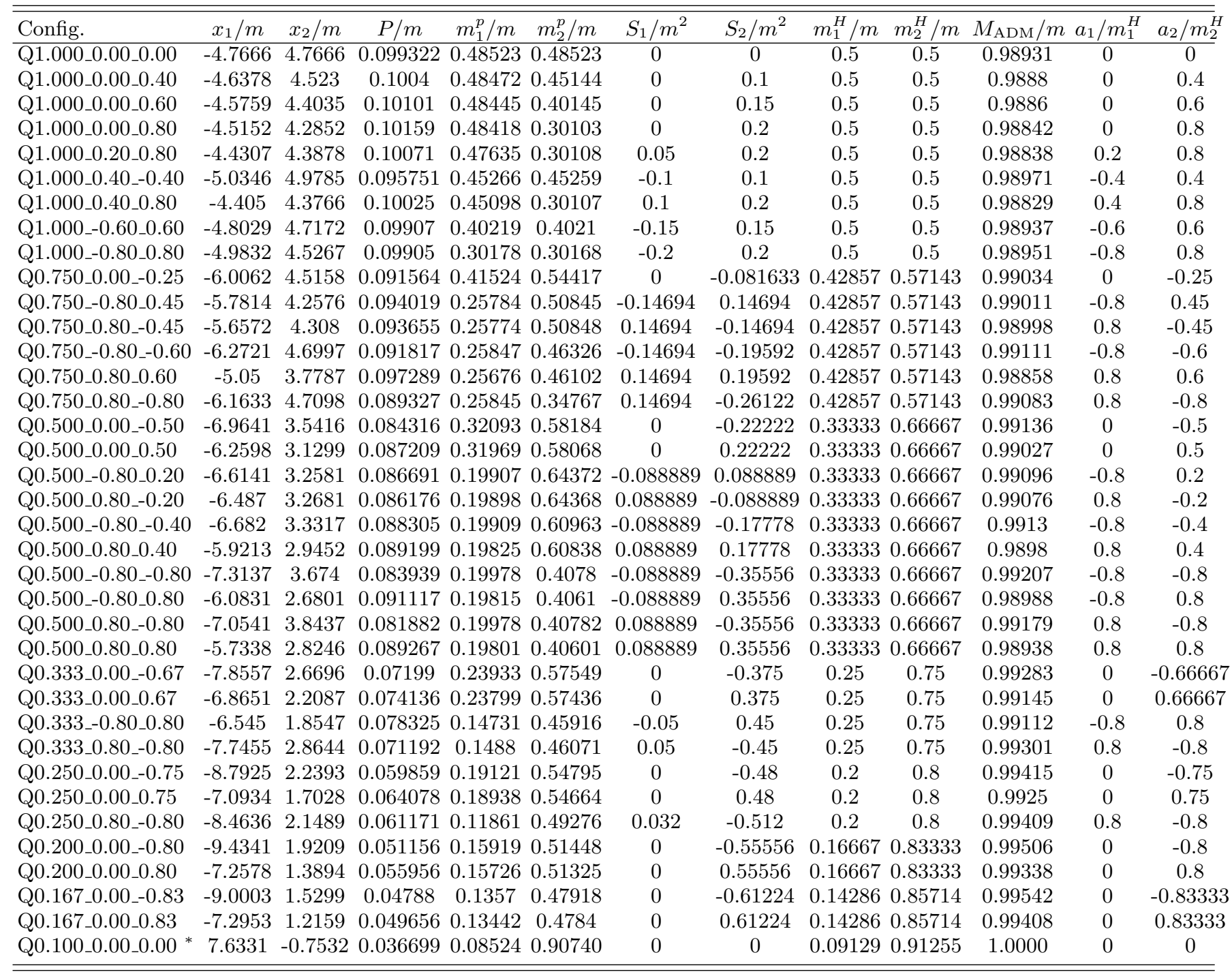

IV. NEW MODELS OF REMNANT MASS, SPIN AND RECOIL

In Refs. [8] and [55] we developed a series expansion for the mass, spin, and recoil velocity of the remnant $\mathrm{BH}$ produced by the merger of a progenitor $\mathrm{BHB}$ with arbitrary $\mathrm{BH}$ spin magnitudes and orientations and arbitrary mass ratio in terms of the variables $\vec{\Delta}, \vec{S}$, and $\delta m$. For the runs presented here, only terms proportional to $S_{\|}, \Delta_{\|}$, and $\delta m$ contribute. In addition, only certain combinations are allowed by symmetry considerations. For more details see Table IV of Ref. [8] and Table VI of Ref. [55]. Here we include all allowed terms up through fourth order. Here we include powers of $\delta m$ when counting orders.
This differs from our previous conventions 8, 55, where we only counted powers in the spin variables and allowed the coefficients of those terms to be arbitrary functions of $\delta m$ (consistent with the symmetries).

The formula for the mass of the remnant $M_{\text {rem }}$ is then 
TABLE II: The mass and spin of the BHBs in Table I after the BHs had time to equilibrate $(t / M=150)$.

\begin{tabular}{|c|c|c|c|c|c|c|c|}
\hline Config. & $m_{1}^{r} / m$ & $m_{2}^{r} / m$ & $\alpha_{1}^{r}$ & $\alpha_{2}^{r}$ & $\delta m_{r}$ & $S_{r} / m_{r}^{2}$ & $\Delta_{r} / m_{r}^{2}$ \\
\hline Q1.000_0.00_0.00 & 0.500001 & 0.500001 & -0.000002 & -0.000002 & 0.000000 & -0.000001 & 0.000000 \\
\hline Q1.000_0.00_0.40 & 0.499998 & 0.500005 & -0.000002 & 0.399919 & -0.000007 & 0.099981 & 0.199962 \\
\hline Q1.000_0.00_0.60 & 0.499998 & 0.499975 & -0.000002 & 0.600030 & 0.000023 & 0.150000 & 0.300009 \\
\hline Q1.000_0.00_0.80 & 0.499999 & 0.499804 & -0.000001 & 0.800605 & 0.000195 & 0.200073 & 0.400225 \\
\hline Q1.000_0.20_0.80 & 0.500006 & 0.499804 & 0.199991 & 0.800585 & 0.000202 & 0.250083 & 0.300196 \\
\hline Q1.000_0.40_-0.40 & 0.500006 & 0.500007 & -0.400013 & 0.399986 & -0.000001 & -0.000006 & 0.399999 \\
\hline Q1.000_0.40_0.80 & 0.500009 & 0.499803 & 0.399974 & 0.800587 & 0.000206 & 0.300099 & 0.200183 \\
\hline Q1.000_-0.60_0.60 & 0.499976 & 0.499980 & -0.600096 & 0.600029 & -0.000004 & -0.000014 & 0.600063 \\
\hline Q1.000_-0.80_0.80 & 0.499801 & 0.499808 & -0.800702 & 0.800576 & -0.000007 & -0.000026 & 0.800639 \\
\hline Q0.750_0.00_-0.25 & 0.428573 & 0.571432 & -0.000002 & -0.250015 & -0.142858 & -0.081638 & -0.142865 \\
\hline Q0.750_-0.80_0.45 & 0.428415 & 0.571439 & -0.800654 & 0.449990 & -0.143045 & -0.000011 & 0.600241 \\
\hline Q0.750_0.80_-0.45 & 0.428417 & 0.571428 & 0.800563 & -0.450042 & -0.143033 & -0.000016 & -0.600235 \\
\hline Q0.750_-0.80_-0.60 & 0.428413 & 0.571397 & -0.800656 & -0.600070 & -0.143011 & -0.343001 & 0.000133 \\
\hline Q0.750_0.80_0.60 & 0.428422 & 0.571391 & 0.800523 & 0.600059 & -0.142995 & 0.342972 & -0.000093 \\
\hline Q0.750_0.80_-0.80 & 0.428412 & 0.571165 & 0.800579 & -0.800777 & -0.142813 & -0.114398 & -0.800692 \\
\hline Q0.500_0.00_-0.50 & 0.333333 & 0.666646 & -0.000003 & -0.500057 & -0.333320 & -0.222243 & -0.333367 \\
\hline Q0.500_0.00_0.50 & 0.333333 & 0.666647 & -0.000003 & 0.500073 & -0.333321 & 0.222250 & 0.333380 \\
\hline Q0.500_-0.80_0.20 & 0.333239 & 0.666684 & -0.800560 & 0.199993 & -0.333471 & -0.000010 & 0.400141 \\
\hline Q0.500_0.80_-0.20 & 0.333239 & 0.666670 & 0.800469 & -0.200003 & -0.333462 & -0.000001 & -0.400120 \\
\hline Q0.500_-0.80_-0.40 & 0.333243 & 0.666681 & -0.800542 & -0.399969 & -0.333463 & -0.266713 & 0.000124 \\
\hline Q0.500_0.80_0.40 & 0.333244 & 0.666666 & 0.800412 & 0.400002 & -0.333452 & 0.266713 & -0.000065 \\
\hline Q0.500_-0.80_-0.80 & 0.333233 & 0.666348 & -0.800574 & -0.800723 & -0.333254 & -0.444809 & -0.266894 \\
\hline Q0.500_-0.80_0.80 & 0.333246 & 0.666358 & -0.800499 & 0.800638 & -0.333244 & 0.266823 & 0.800592 \\
\hline Q0.500_0.80_-0.80 & 0.333232 & 0.666330 & 0.800530 & -0.800798 & -0.333244 & -0.266891 & -0.800708 \\
\hline Q0.500_0.80_0.80 & 0.333245 & 0.666344 & 0.800398 & 0.800701 & -0.333237 & 0.444774 & 0.266924 \\
\hline Q0.333_0.00_-0.67 & 0.249994 & 0.749857 & -0.000002 & -0.666947 & -0.499938 & -0.375127 & -0.500189 \\
\hline Q0.333_0.00_0.67 & 0.249997 & 0.749855 & -0.000004 & 0.666920 & -0.499931 & 0.375108 & 0.500168 \\
\hline Q0.333_-0.80_0.80 & 0.249953 & 0.749624 & -0.800179 & 0.800758 & -0.499883 & 0.400322 & 0.800613 \\
\hline Q0.333_0.80_-0.80 & 0.249944 & 0.749607 & 0.800395 & -0.800865 & -0.499888 & -0.400372 & -0.800748 \\
\hline Q0.250_0.00_-0.75 & 0.200004 & 0.799697 & -0.000002 & -0.750583 & -0.599873 & -0.480297 & -0.600419 \\
\hline Q0.250_0.00_0.75 & 0.199998 & 0.799695 & -0.000003 & 0.750566 & -0.599881 & 0.480291 & 0.600408 \\
\hline Q0.250_0.80_-0.80 & 0.199967 & 0.799568 & 0.800375 & -0.800890 & -0.599880 & -0.480459 & -0.800787 \\
\hline Q0.200_0.00_-0.80 & 0.166673 & 0.832872 & 0.000001 & -0.800897 & -0.666502 & -0.556069 & -0.667348 \\
\hline Q0.200_0.00_0.80 & 0.166665 & 0.832869 & 0.000004 & 0.800884 & -0.666515 & 0.556068 & 0.667342 \\
\hline Q0.167_0.00_-0.83 & 0.142855 & 0.856538 & -0.000002 & -0.834514 & -0.714117 & -0.612992 & -0.715227 \\
\hline Q0.167_0.00_0.83 & 0.142855 & 0.856533 & -0.000001 & 0.834513 & -0.714115 & 0.612989 & 0.715226 \\
\hline
\end{tabular}

given by,

$$
\begin{aligned}
& \frac{M_{\mathrm{rem}}}{m}=\left\{M_{0}+K_{1} \tilde{S}_{\|}+K_{2 a} \tilde{\Delta}_{\|} \delta m+K_{2 b} \tilde{S}_{\|}^{2}+\right. \\
& K_{2 c} \tilde{\Delta}_{\|}^{2}+K_{2 d} \delta m^{2}+K_{3 a} \tilde{\Delta}_{\|} \tilde{S}_{\|} \delta m+ \\
& K_{3 b} \tilde{S}_{\|} \tilde{\Delta}_{\|}^{2}+K_{3 c} \tilde{S}_{\|}^{3}+ \\
& K_{3 d} \tilde{S}_{\|} \delta m^{2}+K_{4 a} \tilde{\Delta}_{\|} \tilde{S}_{\|}^{2} \delta m+ \\
& K_{4 b} \tilde{\Delta}_{\|}^{3} \delta m+K_{4 c} \tilde{\Delta}_{\|}^{4}+K_{4 d} \tilde{S}_{\|}^{4}+ \\
& K_{4 e} \tilde{\Delta}_{\|}^{2} \tilde{S}_{\|}^{2}+K_{4 f} \delta m^{4}+K_{4 g} \tilde{\Delta}_{\|} \delta m^{3}+ \\
& \left.K_{4 h} \tilde{\Delta}_{\|}^{2} \delta m^{2}+K_{4 i} \tilde{S}_{\|}^{2} \delta m^{2}\right\}+ \\
& +\mathcal{O}\left(\epsilon^{5}\right),
\end{aligned}
$$

where $\mathcal{O}\left(\epsilon^{5}\right)$ denotes terms of fifth and higher order in the expansion variables and where variables with tildes are dimensionless, that is $\tilde{S}_{\|}=S_{\|} / m^{2}$ and $\tilde{\Delta}_{\|}=\Delta_{\|} / m^{2}$. As written, Eq. 13 does not reproduce exactly the particle limit since $\delta m \rightarrow \pm 1$ as $\eta \rightarrow 0$. However, we can add terms of order $\mathcal{O}\left(\epsilon^{6}\right)$ and higher to obtain the correct particle limit behavior while simultaneously producing an expansion equivalent to Eq. 113. First, we note that in the particle limit, $M_{\text {rem }}$ is given by $M_{\text {rem }} / m=$ $1+\eta\left(\tilde{E}_{\text {isco }}-1\right)+\mathcal{O}(\eta)^{2}$ (where $m \eta \tilde{E}_{\text {isco }}$ is the energy of a particle at the ISCO). To enforce the particle limit for zero spin we add two terms $K_{6} \delta m^{6}+K_{8} \delta m^{8}$, and then fix the value of these constants by demanding that a reexpansion in terms of $\eta$ gives $1+\eta\left(\tilde{E}_{\text {isco }}^{\text {sch }}-1\right)+\mathcal{O}(\eta)^{2}\left(\tilde{E}_{\text {isco }}^{\text {sch }}\right.$ is the Schwarzschild ISCO energy). We follow a similar procedure for the spin dependent terms. For most terms in Eq. (13), the net effect is to simply multiply the given term by $(4 \eta)^{2}$. The resulting formula for $M_{\text {rem }}$ is given 
TABLE III: Table of the initial orbital frequency $m \omega_{i}$, number of orbits to merger, $N$, and the initial and final eccentricities, $e_{i}$ and $e_{f}$.

\begin{tabular}{lcccc}
\hline \hline Config. & $m \omega_{i}$ & $N$ & $e_{i}$ & $e_{f}$ \\
\hline Q1.000_0.00_0.00 & 0.0300 & 5.3 & 0.028 & 0.005 \\
Q1.000_0.00_0.40 & 0.0313 & 5.4 & 0.021 & 0.006 \\
Q1.000_0.00_0.60 & 0.0320 & 5.6 & 0.021 & 0.005 \\
Q1.000_0.00_0.80 & 0.0327 & 5.7 & 0.020 & 0.004 \\
Q1.000_0.20_0.80 & 0.0324 & 6.2 & 0.020 & 0.002 \\
Q1.000_0.40_-0.40 & 0.0280 & 5.9 & 0.023 & 0.002 \\
Q1.000_0.40_0.80 & 0.0324 & 6.6 & 0.020 & 0.002 \\
Q1.000_-0.60_0.60 & 0.0300 & 5.1 & 0.022 & 0.005 \\
Q1.000_-0.80_0.80 & 0.0300 & 5.1 & 0.022 & 0.005 \\
Q0.750_0.00_-0.25 & 0.0263 & 6.2 & 0.024 & 0.005 \\
Q0.750_-0.80_0.45 & 0.0280 & 5.7 & 0.022 & 0.003 \\
Q0.750_0.80_-0.45 & 0.0280 & 6.1 & 0.023 & 0.006 \\
Q0.750_-0.80_-0.60 & 0.0254 & 4.6 & 0.024 & 0.005 \\
Q0.750_0.80_0.60 & 0.0320 & 7.1 & 0.020 & 0.004 \\
Q0.750_0.80_-0.80 & 0.0250 & 6.6 & 0.025 & 0.005 \\
Q0.500_0.00_-0.50 & 0.0265 & 5.4 & 0.020 & 0.005 \\
Q0.500_0.00_0.50 & 0.0300 & 7.2 & 0.024 & 0.003 \\
Q0.500_-0.80_0.20 & 0.0287 & 5.6 & 0.019 & 0.003 \\
Q0.500_0.80_-0.20 & 0.0287 & 6.2 & 0.019 & 0.005 \\
Q0.500_-0.80_-0.40 & 0.0287 & 4.0 & 0.018 & 0.004 \\
Q0.500_0.80_0.40 & 0.0320 & 6.8 & 0.018 & 0.004 \\
Q0.500_-0.80_-0.80 & 0.0254 & 4.1 & 0.019 & 0.008 \\
Q0.500_-0.80_0.80 & 0.0330 & 6.1 & 0.017 & 0.002 \\
Q0.500_0.80_-0.80 & 0.0250 & 6.1 & 0.021 & 0.005 \\
Q0.500_0.80_0.80 & 0.0330 & 7.8 & 0.017 & 0.002 \\
Q0.333_0.00_-0.67 & 0.0265 & 5.0 & 0.013 & 0.008 \\
Q0.333_0.00_0.67 & 0.0310 & 8.6 & 0.014 & 0.003 \\
Q0.333_-0.80_0.80 & 0.0345 & 7.1 & 0.012 & 0.003 \\
Q0.333_0.80_-0.80 & 0.0260 & 5.3 & 0.014 & 0.006 \\
Q0.250_0.00_-0.75 & 0.0248 & 5.7 & 0.014 & 0.005 \\
Q0.250_0.00_0.75 & 0.0320 & 10.1 & 0.011 & 0.003 \\
Q0.250_0.80_-0.80 & 0.0260 & 5.2 & 0.009 & 0.008 \\
Q0.200_0.00_-0.80 & 0.0238 & 6.4 & 0.015 & 0.006 \\
Q0.200_0.00_0.80 & 0.0325 & 11.3 & 0.011 & 0.002 \\
Q0.167_0.00_-0.83 & 0.0265 & 4.4 & 0.014 & 0.008 \\
Q0.167_0.00_0.83 & 0.0330 & 12.8 & 0.010 & 0.002 \\
\hline \hline
\end{tabular}

by,

$$
\begin{aligned}
& \frac{M_{\mathrm{rem}}}{m}=(4 \eta)^{2}\left\{M_{0}+K_{1} \tilde{S}_{\|}+K_{2 a} \tilde{\Delta}_{\|} \delta m+K_{2 b} \tilde{S}_{\|}^{2}+\right. \\
& K_{2 c} \tilde{\Delta}_{\|}^{2}+K_{2 d} \delta m^{2}+K_{3 a} \tilde{\Delta}_{\|} \tilde{S}_{\|} \delta m+ \\
& K_{3 b} \tilde{S}_{\|} \tilde{\Delta}_{\|}^{2}+K_{3 c} \tilde{S}_{\|}^{3}+ \\
& K_{3 d} \tilde{S}_{\|} \delta m^{2}+K_{4 a} \tilde{\Delta}_{\|} \tilde{S}_{\|}^{2} \delta m+ \\
& K_{4 b} \tilde{\Delta}_{\|}^{3} \delta m+K_{4 c} \tilde{\Delta}_{\|}^{4}+K_{4 d} \tilde{S}_{\|}^{4}+ \\
& K_{4 e} \tilde{\Delta}_{\|}^{2} \tilde{S}_{\|}^{2}+K_{4 f} \delta m^{4}+K_{4 g} \tilde{\Delta}_{\|} \delta m^{3}+ \\
& \left.K_{4 h} \tilde{\Delta}_{\|}^{2} \delta m^{2}+K_{4 i} \tilde{S}_{\|}^{2} \delta m^{2}\right\}+ \\
& {\left[1+\eta\left(\tilde{E}_{\mathrm{ISCO}}+11\right)\right] \delta m^{6},}
\end{aligned}
$$

Here we take $\tilde{E}_{\mathrm{ISCO}}$ from Eq (2.7) of Ref. [56] (we replace the variable $a$ in Ref. [56 with $\alpha_{\text {rem }}$, but similar results are obtained when using $S_{\|} / m^{2}$ instead).
We then verified that the correct leading power of $4 \eta$ in Eq. $(14)$ is indeed 2 by replacing $(4 \eta)^{2}$ with $(4 \eta)^{p}$ and fitting all coefficients and found $p=2.0006$ gives the best fit. In Table VI below, the power $p$ is set to 2 exactly when performing the fits.

To obtain a phenomenological formula for the remnant spin, we follow a similar procedure. Prior to enforcing the particle limit we have,

$$
\begin{array}{r}
\alpha_{\mathrm{rem}}=\frac{S_{\mathrm{rem}}}{M_{\mathrm{rem}}^{2}}=\left\{L_{0}+L_{1} \tilde{S}_{\|}+\right. \\
L_{2 a} \tilde{\Delta}_{\|} \delta m+L_{2 b} \tilde{S}_{\|}^{2}+L_{2 c} \tilde{\Delta}_{\|}^{2}+L_{2 d} \delta m^{2}+ \\
L_{3 a} \tilde{\Delta}_{\|} \tilde{S}_{\|} \delta m+L_{3 b} \tilde{S}_{\|} \tilde{\Delta}_{\|}^{2}+L_{3 c} \tilde{S}_{\|}^{3}+ \\
L_{3 d} \tilde{S}_{\|} \delta m^{2}+L_{4 a} \tilde{\Delta}_{\|} \tilde{S}_{\|}^{2} \delta m+L_{4 b} \tilde{\Delta}_{\|}^{3} \delta m+ \\
L_{4 c} \tilde{\Delta}_{\|}^{4}+L_{4 d} \tilde{S}_{\|}^{4}+L_{4 e} \tilde{\Delta}_{\|}^{2} \tilde{S}_{\|}^{2}+ \\
L_{4 f} \delta m^{4}+L_{4 g} \tilde{\Delta}_{\|} \delta m^{3}+ \\
\left.L_{4 h} \tilde{\Delta}_{\|}^{2} \delta m^{2}+L_{4 i} \tilde{S}_{\|}^{2} \delta m^{2}\right\}+ \\
\mathcal{O}\left(\epsilon^{5}\right) .
\end{array}
$$

Once again, we add higher order terms in order to enforce the correct particle limit behavior. Here the new terms are generated by multiplying the existing terms in Eq. (15) by the next even powers of $\delta m$ that correspond to $\mathcal{O}\left(\epsilon^{5}\right)$ or higher. For instance for the spin independent terms we add $L_{6} \delta m^{6}+L_{8} \delta m^{8}$ and for the linear in the spin terms $L_{5} S_{\|} \delta m^{4}+L_{7} S_{\|} \delta m^{6}$. We then impose the particle limit which is given by $\alpha_{\text {rem }}=$ $\tilde{S}_{\|}+\eta \tilde{J}_{\text {ISCO }}+\mathcal{O}\left(\eta^{2}\right)$. Again, we use Eq (2.8) of Ref. [56] to calculate the ISCO angular momentum, replacing the variable $a$ there with $\alpha_{\text {rem }}$.

After enforcing the particle limit we get,

$$
\begin{array}{r}
\alpha_{\mathrm{rem}}=\frac{S_{\mathrm{rem}}}{M_{\mathrm{rem}}^{2}}=(4 \eta)^{2}\left\{L_{0}+L_{1} \tilde{S}_{\|}+\right. \\
L_{2 a} \tilde{\Delta}_{\|} \delta m+L_{2 b} \tilde{S}_{\|}^{2}+L_{2 c} \tilde{\Delta}_{\|}^{2}+L_{2 d} \delta m^{2}+ \\
L_{3 a} \tilde{\Delta}_{\|} \tilde{S}_{\|} \delta m+L_{3 b} \tilde{S}_{\|} \tilde{\Delta}_{\|}^{2}+L_{3 c} \tilde{S}_{\|}^{3}+ \\
L_{3 d} \tilde{S}_{\|} \delta m^{2}+L_{4 a} \tilde{\Delta}_{\|} \tilde{S}_{\|}^{2} \delta m+L_{4 b} \tilde{\Delta}_{\|}^{3} \delta m+ \\
L_{4 c} \tilde{\Delta}_{\|}^{4}+L_{4 d} \tilde{S}_{\|}^{4}+L_{4 e} \tilde{\Delta}_{\|}^{2} \tilde{S}_{\|}^{2}+ \\
L_{4 f} \delta m^{4}+L_{4 g} \tilde{\Delta}_{\|} \delta m^{3}+ \\
\left.L_{4 h} \tilde{\Delta}_{\|}^{2} \delta m^{2}+L_{4 i} \tilde{S}_{\|}^{2} \delta m^{2}\right\}+ \\
\tilde{S}_{\|}(1+8 \eta) \delta m^{4}+\eta \tilde{J}_{\mathrm{ISCO}} \delta m^{6} .
\end{array}
$$

In order to verify our hypothesis, we first replaced $(4 \eta)^{2}$ with $(4 \eta)^{p}$ and fit for all coefficients in Eq. (16). We find $p=2.015$, which is reasonably close to the expected power of 2 . We then fit again using $p=2$ exactly, and report these fitting parameters in Table VI below.

By using $a=\alpha_{\text {rem }}$ to evaluate the ISCO quantities, the fitting formula for the spin becomes implicit, and the formula for the mass depends directly on the formula for the spin. Therefore, to evaluate the fitting formulas 
TABLE IV: The recoil velocity as calculated using $\ell_{\max }=6$ and $r_{\max }=102.6 \mathrm{~m}$.

\begin{tabular}{|c|c|c|c|c|}
\hline Run & Config. & $V_{x}$ & $V_{y}$ & $V$ \\
\hline 1 & Q1.000_0.00_0.00 & $0.0 \pm 0.0$ & $0.0 \pm 0.0$ & $0.0 \pm 0.0$ \\
\hline 2 & Q1.000_0.00_0.40 & $-24.36 \pm 1.16$ & $-80.37 \pm 0.23$ & $83.97 \pm 0.40$ \\
\hline 3 & Q1.000_0.00_0.60 & $54.72 \pm 1.65$ & $-104.99 \pm 1.92$ & $118.40 \pm 1.87$ \\
\hline 4 & Q1.000_0.00_0.80 & $141.16 \pm 0.52$ & $-39.33 \pm 4.57$ & $146.53 \pm 1.32$ \\
\hline 5 & Q1.000_0.20_0.80 & $-76.92 \pm 2.28$ & $60.74 \pm 0.52$ & $98.01 \pm 1.82$ \\
\hline 6 & Q1.000_0.40_-0.40 & $82.56 \pm 2.39$ & $164.52 \pm 1.12$ & $184.07 \pm 1.47$ \\
\hline 7 & Q1.000_0.40_0.80 & $4.99 \pm 0.84$ & $-53.98 \pm 0.69$ & $54.22 \pm 0.69$ \\
\hline 8 & Q1.000_-0.60_0.60 & $-209.74 \pm 2.12$ & $177.56 \pm 2.07$ & $274.80 \pm 2.10$ \\
\hline 9 & Q1.000_-0.80_0.80 & $-167.03 \pm 3.96$ & $327.50 \pm 1.27$ & $367.63 \pm 2.13$ \\
\hline 10 & Q0.750_0.00_-0.25 & $105.53 \pm 0.49$ & $-86.34 \pm 0.67$ & $136.35 \pm 0.57$ \\
\hline 11 & Q0.750_-0.80_0.45 & $208.49 \pm 0.25$ & $60.82 \pm 6.60$ & $217.18 \pm 1.86$ \\
\hline 12 & Q0.750_0.80_-0.45 & $166.34 \pm 1.33$ & $-271.75 \pm 0.25$ & $318.61 \pm 0.73$ \\
\hline 13 & Q0.750_-0.80_-0.60 & $15.40 \pm 1.42$ & $94.31 \pm 0.79$ & $95.57 \pm 0.81$ \\
\hline 14 & Q0.750_0.80_0.60 & $3.89 \pm 1.57$ & $-27.08 \pm 0.27$ & $27.36 \pm 0.35$ \\
\hline 15 & Q0.750_0.80_-0.80 & $-333.94 \pm 1.70$ & $258.31 \pm 4.36$ & $422.19 \pm 2.99$ \\
\hline 16 & Q0.500_0.00_-0.50 & $155.44 \pm 1.36$ & $222.33 \pm 1.06$ & $271.28 \pm 1.17$ \\
\hline 17 & Q0.500_0.00_0.50 & $-18.50 \pm 1.66$ & $-34.08 \pm 5.12$ & $38.77 \pm 4.56$ \\
\hline 18 & Q0.500_-0.80_0.20 & $62.47 \pm 2.45$ & $66.40 \pm 6.26$ & $91.16 \pm 4.86$ \\
\hline 19 & Q0.500_0.80_-0.20 & $254.30 \pm 0.10$ & $-68.79 \pm 2.32$ & $263.43 \pm 0.61$ \\
\hline 20 & Q0.500_-0.80_-0.40 & $-120.44 \pm 2.83$ & $-128.91 \pm 0.01$ & $176.42 \pm 1.93$ \\
\hline 21 & Q0.500_0.80_0.40 & $-80.19 \pm 0.19$ & $-8.70 \pm 1.76$ & $80.67 \pm 0.26$ \\
\hline 22 & Q0.500_-0.80_-0.80 & $126.69 \pm 1.08$ & $-235.55 \pm 1.65$ & $267.46 \pm 1.54$ \\
\hline 23 & Q0.500_-0.80_0.80 & $59.84 \pm 6.28$ & $142.31 \pm 11.19$ & $154.38 \pm 10.60$ \\
\hline 24 & Q0.500_0.80_-0.80 & $231.96 \pm 0.78$ & $-350.71 \pm 2.17$ & $420.48 \pm 1.86$ \\
\hline 25 & Q0.500_0.80_0.80 & $2.12 \pm 4.77$ & $-0.39 \pm 2.11$ & $2.15 \pm 4.71$ \\
\hline 26 & Q0.333_0.00_-0.67 & $-127.75 \pm 2.03$ & $-257.15 \pm 0.57$ & $287.15 \pm 1.04$ \\
\hline 27 & Q0.333_0.00_0.67 & $23.02 \pm 2.89$ & $-9.61 \pm 1.69$ & $24.94 \pm 2.74$ \\
\hline 28 & Q0.333_-0.80_0.80 & $20.09 \pm 4.71$ & $69.37 \pm 8.74$ & $72.22 \pm 8.50$ \\
\hline 29 & Q0.333_0.80_-0.80 & $346.95 \pm 1.00$ & $21.11 \pm 1.57$ & $347.60 \pm 1.01$ \\
\hline 30 & Q0.250_0.00_-0.75 & $-200.80 \pm 0.04$ & $143.20 \pm 1.07$ & $246.63 \pm 0.62$ \\
\hline 31 & Q0.250_0.00_0.75 & $3.53 \pm 2.91$ & $11.09 \pm 5.46$ & $11.64 \pm 5.27$ \\
\hline 32 & Q0.250_0.80_-0.80 & $254.96 \pm 0.06$ & $-95.67 \pm 1.83$ & $272.32 \pm 0.65$ \\
\hline 33 & Q0.200_0.00_-0.80 & $199.51 \pm 0.62$ & $46.95 \pm 1.27$ & $204.97 \pm 0.67$ \\
\hline 34 & Q0.200_0.00_0.80 & $-1.18 \pm 5.14$ & $-3.20 \pm 1.49$ & $3.41 \pm 2.27$ \\
\hline 35 & Q0.167_0.00_-0.83 & $171.27 \pm 0.72$ & $9.26 \pm 1.27$ & $171.52 \pm 0.73$ \\
\hline 36 & Q0.167_0.00_0.83 & $3.33 \pm 3.27$ & $0.51 \pm 0.29$ & $3.37 \pm 3.23$ \\
\hline
\end{tabular}

for any given initial binary, we use a rapidly converging iterative process where the initial $a$ is set to $S_{\|} / \mathrm{m}^{2}$.

Finally, we fit the recoil to the formula,

$$
\begin{gathered}
v_{\perp}=H \eta^{2}\left(\tilde{\Delta}_{\|}+H_{2 a} \tilde{S}_{\|} \delta m+H_{2 b} \tilde{\Delta}_{\|} \tilde{S}_{\|}+H_{3 a} \tilde{\Delta}_{\|}^{2} \delta m\right. \\
+H_{3 b} \tilde{S}_{\|}^{2} \delta m+H_{3 c} \tilde{\Delta}_{\|} \tilde{S}_{\|}^{2}+H_{3 d} \tilde{\Delta}_{\|}^{3}+H_{3 e} \tilde{\Delta}_{\|} \delta m^{2} \\
+H_{4 a} \tilde{S}_{\|} \tilde{\Delta}_{\|}^{2} \delta m+H_{4 b} \tilde{S}_{\|}^{3} \delta m+H_{4 c} \tilde{S}_{\|} \delta m^{3} \\
\left.+H_{4 d} \tilde{\Delta}_{\|} \tilde{S}_{\|} \delta m^{2}+H_{4 e} \tilde{\Delta}_{\|} \tilde{S}_{\|}^{3}+H_{4 f} \tilde{S}_{\|} \tilde{\Delta}_{\|}^{3}\right)(17) \\
\tilde{\xi}=a+b \tilde{S}_{\|}+c \delta m \tilde{\Delta}_{\|},
\end{gathered}
$$

where we have added a leading power of $\eta^{2}$ to the expansion. The issue of the leading power of $\eta$ for the recoil was discussed in the context of the off-plane recoils in Ref. [12, 37, where the possibility of a leading $\eta^{3}$ versus $\eta^{2}$ was studied with full numerical simulations. Further study of recoils in the small mass ratio perturbative regime [57] led to the conclusion that the terms of the recoil linear in the spin should scale as $\eta^{2}$ and postNewtonian expansions including quadratic terms in the spin also show a leading $\eta^{2}$ behavior 40] (again for low eccentricity, in-plane orbits).

Finally, we tested the leading $\eta^{2}$ dependence in Eq. (17) by allowing the power of $\eta$ to be free. Interestingly, we do not find $p=2$, but rather we find that the minimum in the fit is quite shallow with similar results for interval $1.5 \leq p \leq 2.5$ (the minimum is at $p \sim 2.29$ ). Since $p=2$ gives the correct particle limit behavior for quasicircular orbits (at least at moderate mass ratios, see though Refs. [58, 59] for a discussion on resonance recoil which scale as $\eta^{1.5}$ ), we enforce $p=2$ for the fits presented in Table VI below.

We note that a factor of $\eta^{p}$ in the coefficients is not independent from the expansion proposed in Refs. 8] and [55] since $4 \eta=1-\delta m^{2}$ and this allows us to recast all powers of $\eta$ into the original form of the expansion.

The 17 constants in Eqs. (17) and (18), 19 constants in Eq. (14), and 19 constants in Eq. (16) were obtained 
TABLE V: The final remnant mass and spin as measured using the IH formalism and as measured from the radiation of energy and angular momentum.

\begin{tabular}{|c|c|c|c|c|c|}
\hline Run & Config. & $\delta \mathcal{M}^{I H}$ & $\delta \mathcal{M}^{\text {rad }}$ & $\alpha_{\mathrm{rem}}^{I H}$ & $\alpha_{\mathrm{rem}}^{r a d}$ \\
\hline 1 & Q1.000_0.00_0.00 & $0.048379 \pm 0.000001$ & $0.047937 \pm 0.000177$ & $0.686419 \pm 0.000007$ & $0.685034 \pm 0.004747$ \\
\hline 2 & Q1.000_0.00_0.40 & $0.054557 \pm 0.000002$ & $0.053888 \pm 0.000190$ & $0.745985 \pm 0.000058$ & $0.745180 \pm 0.004325$ \\
\hline 3 & Q1.000_0.00_0.60 & $0.058316 \pm 0.000004$ & $0.057438 \pm 0.000250$ & $0.774671 \pm 0.000195$ & $0.774270 \pm 0.004519$ \\
\hline 4 & Q1.000_0.00_0.80 & $0.062821 \pm 0.000007$ & $0.061610 \pm 0.000362$ & $0.802619 \pm 0.000086$ & $0.802453 \pm 0.004977$ \\
\hline 5 & Q1.000_0.20_0.80 & $0.067692 \pm 0.000001$ & $0.066171 \pm 0.000443$ & $0.830671 \pm 0.000015$ & $0.830647 \pm 0.005519$ \\
\hline 6 & Q1.000_0.40_-0.40 & $0.048437 \pm 0.000000$ & $0.047998 \pm 0.000163$ & $0.685844 \pm 0.000001$ & $0.683873 \pm 0.005278$ \\
\hline 7 & Q1.000_0.40_0.80 & $0.073515 \pm 0.000003$ & $071532 \pm 0.000572$ & $0.857465 \pm 0.000046$ & $0.857999 \pm 0.006244$ \\
\hline 8 & Q1.000_-0.60_0.60 & $0.048780 \pm 0.000000$ & $0.048268 \pm 0.000202$ & $0.685258 \pm 0.000000$ & $0.683850 \pm 0.004611$ \\
\hline 9 & Q1.000_-0.80_0.80 & $0.049353 \pm 0.000000$ & $0.048593 \pm 0.000304$ & $0.684235 \pm 0.000020$ & $0.682995 \pm 0.005103$ \\
\hline 10 & Q0.750_0.00_-0.25 & $0.042681 \pm 0.000009$ & $0.042368 \pm 0.000179$ & $0.621171 \pm 0.000025$ & $0.618973 \pm 0.006118$ \\
\hline 11 & Q0.750_-0.80_0.45 & $0.046525 \pm 0.000001$ & $045794 \pm 0.000280$ & $0.685173 \pm 0.000021$ & $0.684202 \pm 0.005942$ \\
\hline 12 & Q0.750_0.80_-0.45 & $0.047283 \pm 0.000001$ & $046683 \pm 0.000270$ & $0.662124 \pm 0.000027$ & $0.660424 \pm 0.006072$ \\
\hline 13 & Q0.750_-0.80_-0.60 & $0.033808 \pm 0.000000$ & $33435 \pm 0.000204$ & $0.451036 \pm 0.000001$ & $0.449575 \pm 0.005390$ \\
\hline 14 & Q0.750_0.80_0.60 & $0.075774 \pm 0.000000$ & $0.073304 \pm 0.000761$ & $0.871698 \pm 0.000003$ & $0.872990 \pm 0.007658$ \\
\hline 15 & Q0.750_0.80_-0.80 & $0.042577 \pm 0.000000$ & $0.042037 \pm 0.000329$ & $0.586122 \pm 0.000000$ & $0.584030 \pm 0$. \\
\hline 16 & Q0.500_0.00_-0.50 & $0.031757 \pm 0.000001$ & $0.031607 \pm 0.000107$ & $69 \pm 0.000001$ & $0.458375 \pm$ \\
\hline 17 & Q0.500_0.00_0.50 & $0.050577 \pm 0.000012$ & $0.049510 \pm 0.000268$ & $0.778577 \pm 0.000018$ & $0.778307 \pm 0.004860$ \\
\hline 18 & Q0.500_-0.80_0.20 & $0.038377 \pm 0.000001$ & $0.037656 \pm 0.000195$ & $0.638918 \pm 0.000018$ & $0.638885 \pm 0.004488$ \\
\hline 19 & Q0.500_0.80_-0.20 & $0.039610 \pm 0.000013$ & $9003 \pm$ & $13 \pm 0.000022$ & $0.605347 \pm 0.0$ \\
\hline 20 & Q0.500_-0.80_-0.40 & $0.030257 \pm 0.000001$ & $29824 \pm 0.000142$ & $54 \pm 0.000004$ & $0.441744 \pm 0.0$ \\
\hline 21 & Q0.500_0.80_0.40 & $0.054693 \pm 0.000002$ & $0.053274 \pm 0.000363$ & $0.790499 \pm 0.000012$ & $0.791070 \pm 0.005010$ \\
\hline 22 & Q0.500_-0.80_-0.80 & $0.026965 \pm 0.000001$ & $0.026574 \pm 0.000175$ & $0.305299 \pm 0.000000$ & $0.304648 \pm 0.004239$ \\
\hline 23 & Q0.500_-0.80_0.80 & $4259 \pm 0.000001$ & $2322 \pm 0.0005$ & $3813 \pm 0.000016$ & $0.825549 \pm 0$ \\
\hline 24 & Q0.500_0.80_-0.80 & $0.031687 \pm 0.000001$ & $1281 \pm 0.000219$ & $368 \pm 0.000001$ & $0.408069 \pm 0.005900$ \\
\hline 25 & Q0.500_0.80_0.80 & $0.075669 \pm 0.000021$ & $0.071617 \pm 0.001144$ & $0.902719 \pm 0.000354$ & $0.906457 \pm 0.008499$ \\
\hline 26 & Q0.333_0.00_-0.67 & $0.021506 \pm 0.000006$ & $0.021379 \pm 0.000114$ & $0.240088 \pm 0.000006$ & $0.239368 \pm 0.003257$ \\
\hline 27 & Q0.333_0.00_0.67 & $0.045862 \pm 0.000010$ & & $71 \pm 0.000$ & $0.825458 \pm 0$ \\
\hline 28 & Q0.333_-0.80_0.80 & $0.047937 \pm 0.000014$ & $0.046294 \pm 0.000398$ & $25 \pm 0.000188$ & $0.857221 \pm 0.004083$ \\
\hline 29 & Q0.333_0.80_-0.80 & $0.022026 \pm 0.000004$ & $0.021768 \pm 0.000115$ & $0.206316 \pm 0.000002$ & $0.205397 \pm 0.003613$ \\
\hline 30 & Q0.250_0.00_-0.75 & $0.016007 \pm 0.000001$ & $.015923 \pm 0.000101$ & $0.067207 \pm 0.000000$ & $0.066201 \pm 0.002989$ \\
\hline 31 & Q0.250_0.00_0.75 & $0.041023 \pm$ & $639 \pm$ & $368 \pm 0$ & $0.855422 \pm 0.004317$ \\
\hline 32 & Q0.250_0.80_-0.80 & $0.016470 \pm 0.000000$ & $16191 \pm 0.000100$ & $0.057516 \pm 0.000001$ & $0.057117 \pm 0.002698$ \\
\hline 33 & Q0.200_0.00_-0.80 & $0.012631 \pm 0.000007$ & $0.012556 \pm 0.000091$ & $-0.067330 \pm 0.000001$ & $-0.067585 \pm 0.002660$ \\
\hline 34 & Q0.200_0.00_0.80 & $0.036968 \pm 0.000044$ & $0.034071 \pm 0.000500$ & $0.872432 \pm 0.000480$ & $0.876983 \pm 0.004144$ \\
\hline 35 & Q0.167_0.00_-0.83 & $0.010495 \pm 0.000004$ & $0.010396 \pm 0.000067$ & $-0.172301 \pm 0.000003$ & $-0.172286 \pm 0.001438$ \\
\hline 36 & Q0.167_0.00_0.83 & $0.033350 \pm 0.000003$ & $0.030324 \pm 0.000367$ & $0.888377 \pm 0.000166$ & $0.893634 \pm 0.003402$ \\
\hline 37 & Q0.100_0.00_0.00 & $0.0044 \pm 0.0001$ & & $0.261 \pm 0.002$ & \\
\hline
\end{tabular}
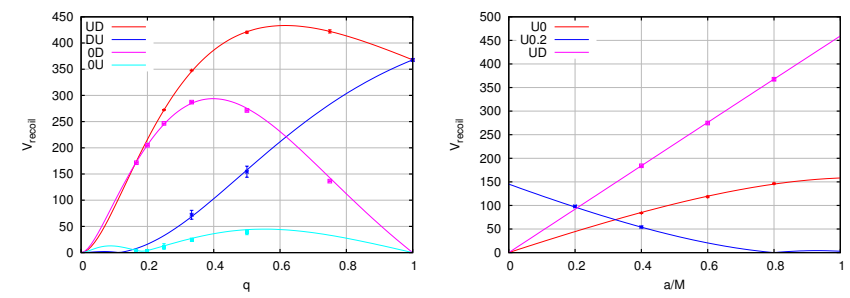

FIG. 4: The recoils for the families UD/DU/0U/0D and for equal masses cases as given in Table $\mathrm{I}$

by a least-squares fit to the results of our 36 simulations and, in the case of the fits of the final mass and spin, additional 38 SXS runs [60] and a $q=1: 10$ simulation. Note that, as explained above, we allow $v_{\perp}$ to be positive and negative and thereby allow $\tilde{\xi}$ to be continuous (See Fig. 7).
The results of the recoil velocity fit and residuals to the entire set of 36 runs is shown in Fig. 5. We observe that the residuals are below $7 \mathrm{~km} \mathrm{~s}^{-1}$.

In order to assess the accuracy of our formula, we compare its predictions for 8 independent runs from the group at AEI reported in Ref. 61 and 16 from the SXS collaboration in Ref. 60]. The results are shown in Fig. 6. We observe that while the residuals of our runs and those of AEI are similar and relatively small (i.e., within $10 \mathrm{~km} \mathrm{~s}^{-1}$ ), the residuals with respect to the SXS runs are roughly 3 times larger. We note that while in this paper (and Ref. 61]) recoils are computed using the radiated linear momentum, the SXS catalog reports coordinate velocities.

Note that while we use the recoils of Ref. 61 as an independent test of our fitting formula, the recoil formula proposed in 61] [Eq. (42) there] does not respect the symmetry of exchange of black hole labels $1 \leftrightarrow 2$, hence 


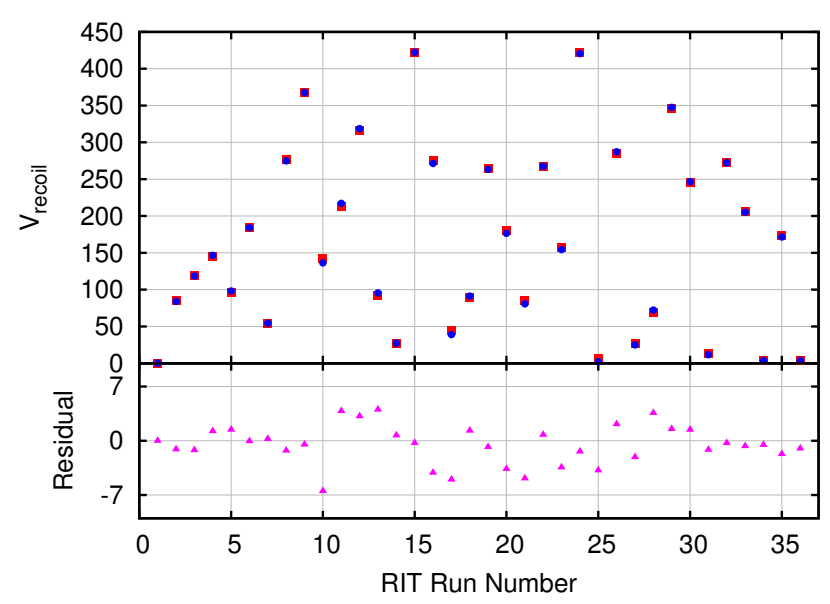

FIG. 5: Fit and residuals to 36 RIT data (See Table IV with a root mean square deviation, $\mathrm{RMS}=2.5 \mathrm{~km} \mathrm{~s}^{-1}$

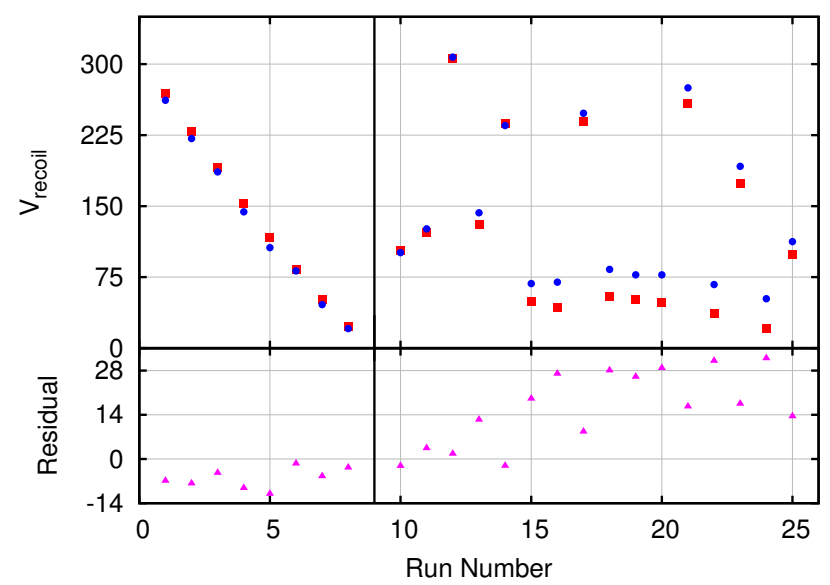

FIG. 6: Recoil Fit and residuals to 8 AEI $\left(\mathrm{RMS}=6.8 \mathrm{~km} \mathrm{~s}^{-1}\right)$ simulations and $16 \mathrm{SXS}\left(\mathrm{RMS}=20 \mathrm{~km} \mathrm{~s}^{-1}\right)$ simulations.

we would expect it to be less accurate outside of the region of parameter space used to generate that fit.

Interestingly, by allowing $v_{\perp}$ in Eq. (12) to take on positive and negative values, the angle $\tilde{\xi}$ can be restricted to the interval $90^{\circ} \leq \tilde{\xi} \leq 180^{\circ}$ and its average value, as shown in Fig. 7 , is $\tilde{\xi} \sim 148^{\circ}$, which is very close to the estimate $\tilde{\xi} \sim 145^{\circ}$ in Ref. [38. Note that the dispersion

We use a similar procedure to fit the final remnant mass and spin to Eqs. (14) and (16). Here, however, we add the data from the SXS catalog [60] (which include results from highly spinning BHBs) into our fits, as well as results from a non-spinning binary with mass ratio $q=$ $1 / 10$ from Refs. 63, 64]. The resulting fitting parameters are given in Table VI. The data, fit, and residuals for the remnant spins are shown in Figs. 8 and 9 The residuals is quite large though. As part of our fitting of the recoil, we must simultaneously fit $\tilde{\xi}$ to Eq. 18p. Interestingly, the choice of coefficients in Eq. (18) that optimizes the fit is close to $c / b=3 / 7$. This ratio is significant because the effective spin defined in Ref. [62] is given by $\vec{S}_{\text {effective }}=$ $\vec{S}+\frac{3}{7} \vec{\Delta} \delta m$. Thus it appears that the functional form of $\tilde{\xi}$ that minimizes the residuals is essentially a linear function in the effective spin.

The fitted values of all coefficients in Eqs. 17) and (18), as well as the uncertainties in these values are given in Table VI. We estimate the errors in the fitting parameters by adding Gaussian

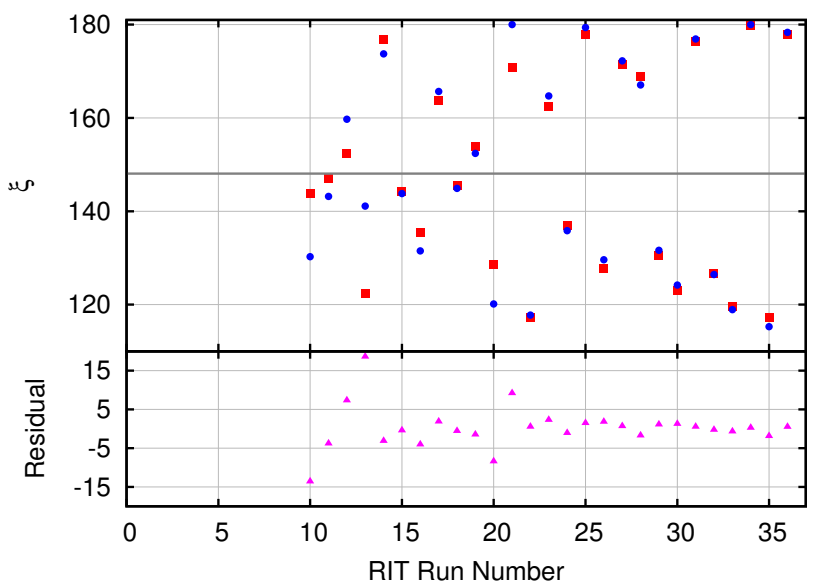

FIG. 7: The angle $\tilde{\xi}$ as defined in Eq. 18, computed from the measured magnitude of the recoil (circles), compared to the fitted formula (squares). The angle is not defined for the (first) 9 equal mass runs in Table IV. The average of $\tilde{\xi} \sim 148^{\circ}$ (gray line) is near the previously measured value of $\tilde{\xi} \sim 145^{\circ}$, but the new spin-dependent formula for $\tilde{\xi}$ significantly reduces the residuals.

distributed random noise to the fitting function. Each data point is given a different random number, and the width of the Gaussian is determined by the estimated error in that data. By performing the fit 50,000 times, each with a different set of noise, a distribution in the fitting parameters is found. The standard deviation of these distributions is recorded as the error in the fitting parameter in Table VI.

are below $6 \times 10^{-4}$

We can compare the residuals of our fit with other fitting formulas in the literature, for instance, for the final spin of the merged black hole given in Ref. 65] (we denote this fit by AEI). The results are shown in Fig. 10 for the current data (RIT) and the SXS data. We observe a clear improvement of our fitting formula (16), with over an order of magnitude reduction in the residuals.

We see that our fitting formula for the final remnant 
TABLE VI: Table of fitting parameters for recoil, mass, and spin formulas.

\begin{tabular}{lc|c|ccc}
\hline \hline H & $7367.250029 \pm 66.122336$ & M0 & $0.951507 \pm 0.000030$ & L0 & $0.686710 \pm 0.000039$ \\
H2a & $-1.626094 \pm 0.053888$ & K1 & $-0.051379 \pm 0.000193$ & L1 & $0.613247 \pm 0.000168$ \\
H2b & $-0.578177 \pm 0.055790$ & K2a & $-0.004804 \pm 0.000514$ & L2a & $-0.145427 \pm 0.000473$ \\
H3a & $-0.717370 \pm 0.077605$ & K2b & $-0.054522 \pm 0.000690$ & L2b & $-0.115689 \pm 0.000761$ \\
H3b & $-2.244229 \pm 0.137982$ & K2c & $-0.000022 \pm 0.000010$ & L2c & $-0.005254 \pm 0.000332$ \\
H3c & $-1.221517 \pm 0.176699$ & K2d & $1.995246 \pm 0.000497$ & L2d & $0.801838 \pm 0.000514$ \\
H3d & $-0.002325 \pm 0.021612$ & K3a & $0.007064 \pm 0.002680$ & L3a & $-0.073839 \pm 0.002986$ \\
H3e & $-1.064708 \pm 0.133021$ & K3b & $-0.017599 \pm 0.001678$ & L3b & $0.004759 \pm 0.001374$ \\
H4a & $-0.579599 \pm 0.297351$ & K3c & $-0.119175 \pm 0.001054$ & L3c & $-0.078377 \pm 0.000911$ \\
H4b & $-0.455986 \pm 0.302432$ & K3d & $0.025000 \pm 0.001951$ & L3d & $1.585809 \pm 0.001777$ \\
H4c & $0.010963 \pm 0.174289$ & K4a & $-0.068981 \pm 0.004251$ & L4a & $-0.003050 \pm 0.001910$ \\
H4d & $1.542924 \pm 0.274459$ & K4b & $-0.011383 \pm 0.001709$ & L4b & $-0.002968 \pm 0.001431$ \\
H4e & $-4.735367 \pm 0.430869$ & K4c & $-0.002284 \pm 0.000192$ & L4c & $0.004364 \pm 0.000532$ \\
H4f & $-0.284062 \pm 0.174087$ & K4d & $-0.165658 \pm 0.003100$ & L4d & $-0.047204 \pm 0.003250$ \\
$\mathrm{a}$ & $2.611988 \pm 0.028327$ & K4e & $0.019403 \pm 0.003220$ & L4e & $-0.053099 \pm 0.003682$ \\
$\mathrm{~b}$ & $1.383778 \pm 0.092915$ & K4f & $2.980990 \pm 0.001197$ & L4f & $0.953458 \pm 0.001210$ \\
$\mathrm{c}$ & $0.549758 \pm 0.113300$ & K4g & $0.020250 \pm 0.002524$ & L4g & $-0.067998 \pm 0.002369$ \\
& & K4h & $-0.004091 \pm 0.002057$ & L4h & $0.001629 \pm 0.000980$ \\
& & K4i & $0.078441 \pm 0.003263$ & L4i & $-0.066693 \pm 0.003289$ \\
\hline \hline
\end{tabular}

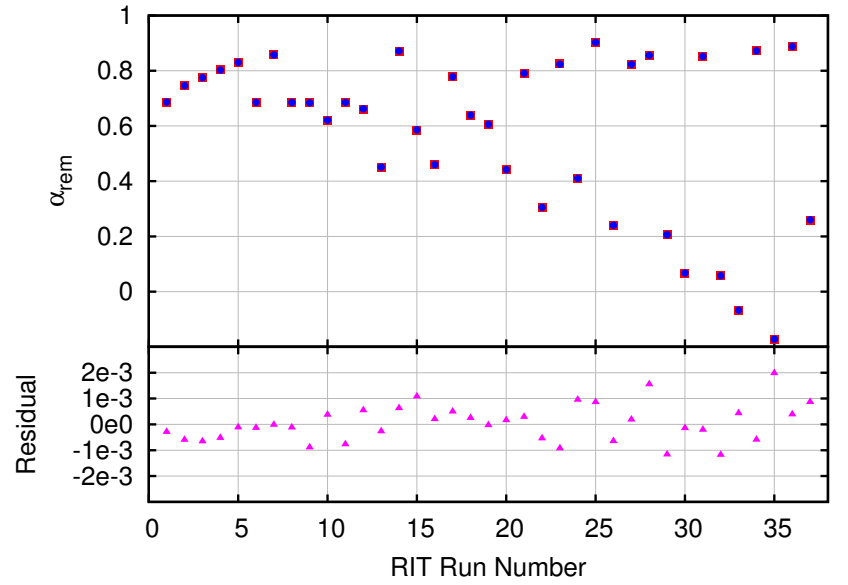

FIG. 8: Fit to the remnant spin using RIT+SXS data and residuals of RIT runs as labeled by run number (see Table V). $\mathrm{RMS}=7.16 \times 10^{-4}$.

spin is remarkably accurate over a wide range of mass ratios. In addition, we constructed the formula such that it gives the correct small-mass limit behavior. We thus expect that our formula will be reasonably accurate for all mass ratios, at least for moderate spins $(\alpha \leq 0.9)$.

A similar analysis for the final remnant mass is shown in Figs. 11, 12, and 13. Once again we used the SXS data in generating our fits. Here we see residuals of order $3 \times 10^{-4}$ for our new formula and residuals several times larger for the AEI formula [66].

An interesting consequence of the form of the recoil velocity (17) is that, for certain combinations of the spins and the mass ratio the total magnitude can be very small. In Fig. 14, we plot the values of $\alpha_{2}$ and $q$ that lead to small recoils for a given $\alpha_{1}$. Apart from the zero recoil imposed by symmetry, i.e. $q \rightarrow 0$ and $q=1, \alpha_{1}=\alpha_{2}$,

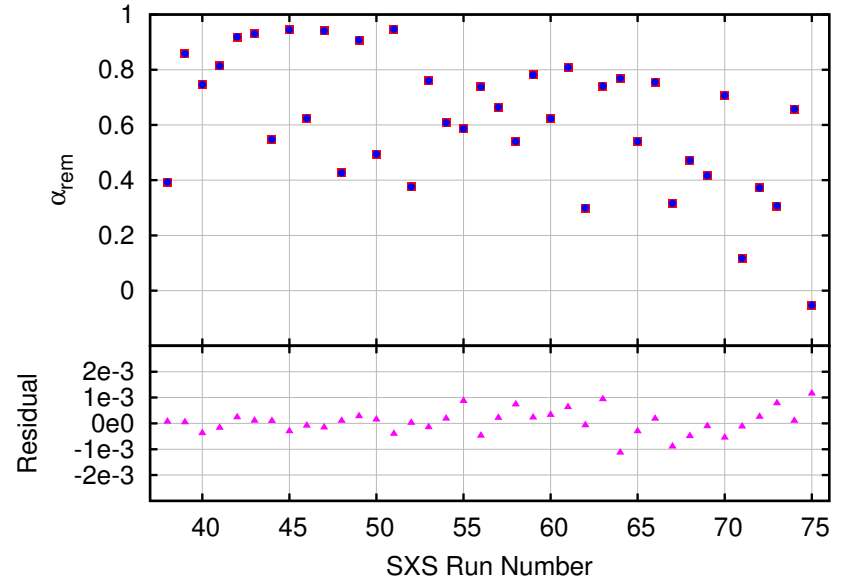

FIG. 9: Fit to the remnant spin using RIT+SXS data residuals for the SXS data. $\mathrm{RMS}=4.73 \times 10^{-4}$.
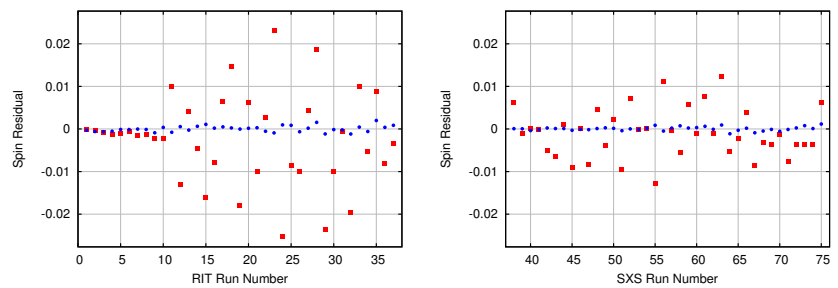

FIG. 10: A comparison of the residuals from our spin fit and the AEI fit for the RIT data (left) and SXS data (right). Residuals from our formula are denoted by (blue) circles and residuals from AEI formula are denoted by (red) squares.

there appear to be two branches that lead to vanishing recoils. One branch spans all mass ratios with $0.6 \lesssim \alpha_{2} \lesssim$ 0.75 and the other branch only spans the smaller mass ratio regime $q \lesssim 0.4$ and larger spins $0.74 \lesssim \alpha_{2} \leq 1$. 


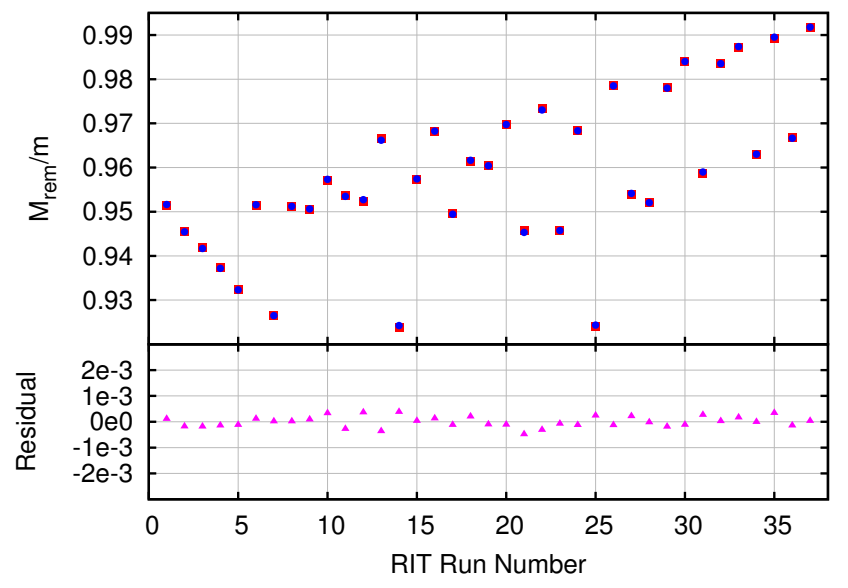

FIG. 11: Predicted and measured remnant mass for RIT data versus run number. $\mathrm{RMS}=2.07 \times 10^{-4}$.

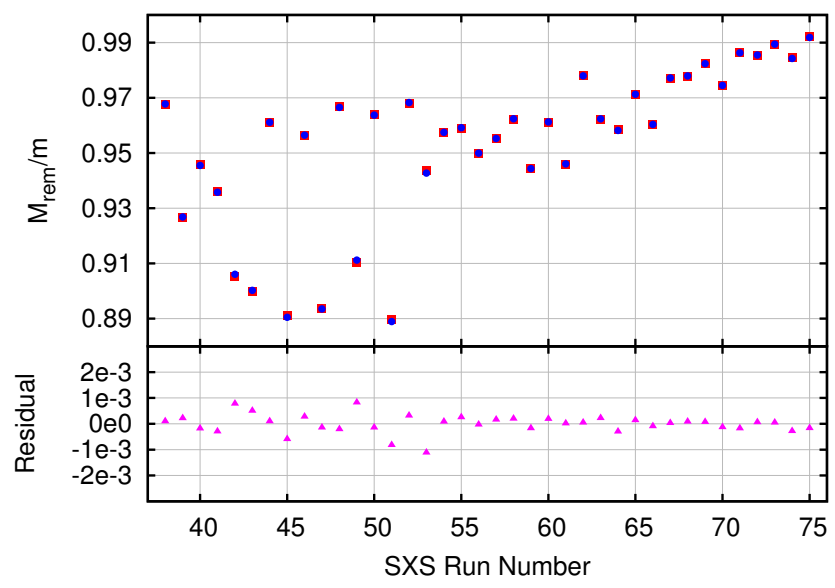

FIG. 12: Predicted and measured remnant mass for the SXS data. $\mathrm{RMS}=3.56 \times 10^{-4}$.

Interestingly, the vanishing of the recoil velocity does not arise from any symmetry, but rather from a cancellation of processes that involve a wobbling of the center of mass as the BHB slowly inspirals, the recoil generated during the rapid plunge, and the post-merger anti-kick 67, which is generated during the ring-down phase. All of these three stages combine to produce a non moving
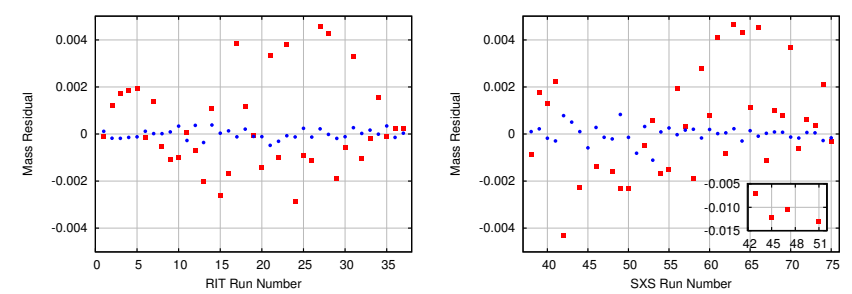

FIG. 13: Residuals for our mass fit (small blue circles) to the RIT (left) and SXS data (right) compared to the AEI fitting formula (large red squares).

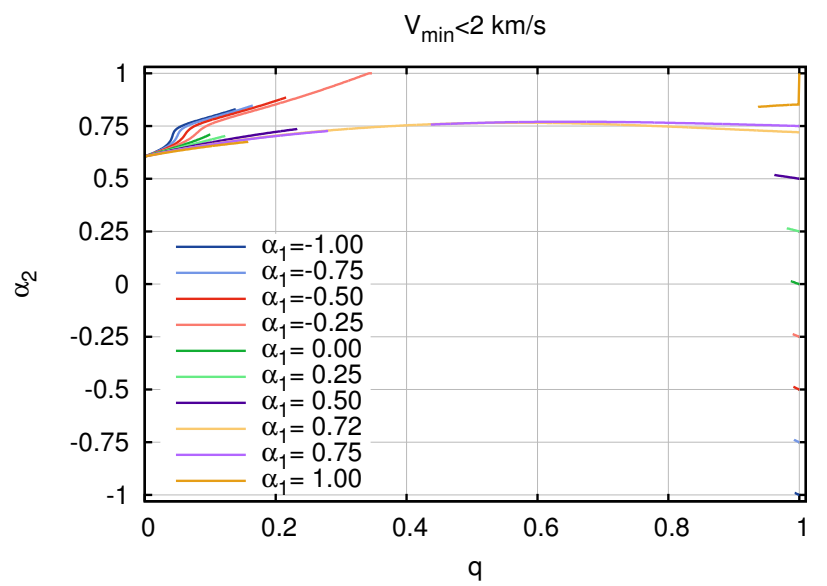

FIG. 14: The BHB configurations that lead to a final remnant black hole with zero recoil. Additions zero recoils exist for $q=0$ and for $q=1$ with $\alpha_{1}=\alpha_{2}$.

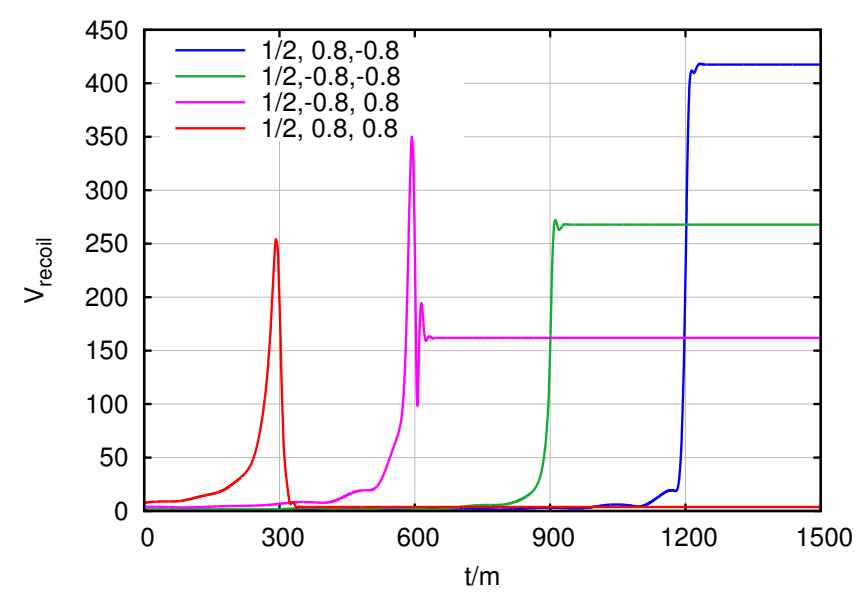

FIG. 15: The radiated linear momentum for the Q0.500_0.80_0.80 configuration (red). We observe the wobbling from the inspiral, a sudden raise to above $250 \mathrm{~km} \mathrm{~s}^{-1}$ due to the merger, and the final antikick from the ringdown phase which reduces the final velocity of the remnant to $2 \mathrm{~km} \mathrm{~s}^{-1}$. For comparison we also show the Q0.500 $\pm 0.80_{-} \pm 0.80$ configurations to see the different effects of the superposition of spin (signs) and unequal mass components of the recoil.

final $\mathrm{BH}$, but in the process the $\mathrm{BH}$ is displaced from the original center of mass of the binary. In Fig. 15 we provide an explicit example for run \#25 Q0.500_0.80_0.80 of a near zero final recoil.

It is interesting to recall here the zero-recoil superkicks seen in 6, 68. In that case the bobbing of the BHs up and down can be tuned by choosing the azimuthal orientation of the spin such that the merger occurs when the bobbing velocity is instantaneously zero, which leads to a vanishing recoil.

In Fig. 15 we show the velocity of the center of mass versus time for the four possible combinations of signs of the spins for a $q=1 / 2$ binary with spin magnitudes 


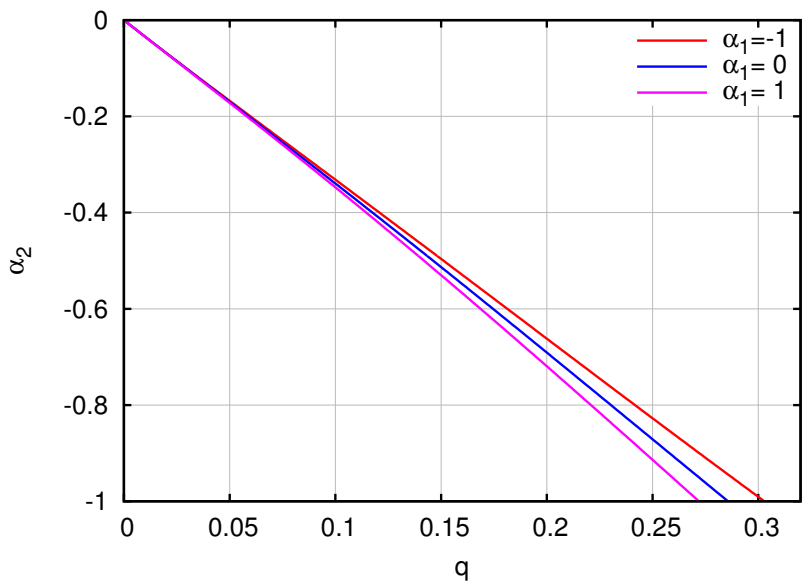

FIG. 16: The BHB configurations that lead to a final Schwarzschild black hole. We note that the spin of the small hole has little influence on the values of the mass ratio and spin of the large black hole that lead to a vanishing final spin.

$\alpha_{i}=0.8\left(\mathrm{Q} 0.500_{-} \pm 0.80_{-} \pm 0.80\right)$. The UD configuration Q0.500_0.80_-0.80 recoils at $420 \mathrm{~km} \mathrm{~s}^{-1}$ while the DD configuration recoils at a more modest $267 \mathrm{~km} \mathrm{~s}^{-1}$. Reversing the spin directions for both $\mathrm{BH}$ leads to a DU configuration that recoils at $154 \mathrm{~km} \mathrm{~s}^{-1}$. Finally, a UU configuration recoils at a very small $2 \mathrm{kms}^{-1}$. Notably, the recoil of the DU configuration agrees with the purely unequal mass recoil $v_{m}(q=1 / 2)$, and the difference between the recoil velocities of the UD and DU configurations, as well as the differences in the recoil between the DD and UU configurations are both around $266 \mathrm{~km} \mathrm{~s}^{-1}$.

It is also interesting to see which configurations lead to a remnant with vanishing spin (as was done in Ref. 69], Fig 4a). Our results are shown in Fig. 16. They show that in order to have a final Schwarzschild black hole the larger hole must be counteraligned with the orbital angular momentum and the smaller hole must bear a mass ratio less than 0.3 . This small mass ratio also explains the relatively weak dependence on the spin of the secondary black hole.

In the equal-mass regime, Eq. (14) predicts a maximum amount of radiated energy of $\overline{M_{\text {rem }}^{\max }} / m=0.88693 \pm$ 0.00027 , i.e., a maximum radiated energy of $11.3 \%$, and Eq. (16) predicts a maximum remnant spin of $\alpha_{\mathrm{rem}}^{\max }=$ $0.95166 \pm 0.00027$, both of which closely agree with the predictions of Ref. [70].

\section{STATISTICAL DISTRIBUTIONS}

In order to visualize the consequences of Eqs. (14, (16), (17), and (18), we study the distributions of recoils and remnant masses and spins from BHBs where the individual $\mathrm{BH}$ spins are either aligned or counteraligned with the orbital angular momentum. We study 9 families of distributions of progenitors: both BH spins aligned with the orbital angular momentum, both coun- teraligned, two families where one $\mathrm{BH}$ is aligned and the other counter aligned, four families where one $\mathrm{BH}$ spin direction is chosen randomly (aligned or counteraligned) and the other direction is fixed, and one family where both $\mathrm{BH}$ orientations are chosen randomly. In all cases the spin-magnitudes are chosen from the cold accretion distribution in [7, which is represented by $P(\alpha)=(1-\alpha)^{b-1} \alpha^{a-1}$, where $a=5.935$ and $b=1.856$, and the mass ratio distribution from 71-73, which is given by $P(q) \propto q^{-0.3}(1-q)$. In Figs. 1718 , and 19 we show the probabilities for a remnant recoiling with speed $v$, having mass $M_{\text {rem }}$, and spin $\alpha_{\text {rem }}$.

There are several interesting things to note from Fig. 17. First, the probability for large recoils is much larger for the UD family of configurations than for any of the other (nonrandom) configurations. The UR and $\mathrm{RD}$ (here $\mathrm{R}$ denotes that the spin orientation is chosen randomly) families both show the same probabilities at high velocities. The reason for this is that high velocities can only come from a UD type configuration. Both the UR and RD families have a $50 \%$ probability for a given configuration to be UD.

From Fig. 18, we see that the UU families show significant probabilities for smaller remnant mass. Small remnant masses occur for near-equal-mass UU systems, as this maximizes the radiated energy. The UR, RU, and RR families show a similar tail at smaller remnant masses. The UR and RU configurations have a $50 \%$ probability of being UU and the RR configurations have a $25 \%$ probability of being UU. Hence the $P(m)$ for the RR configurations is half the value of $P(m)$ for the UR and RU families.

Finally, in Fig. 19, we see that the probability for a final remnant spin counteraligned with the binary's orbital angular momentum is nearly equal for the DD and UD families. The reason is this can only happen in the small mass ratio regime with a counteralign larger BH. Similarly the UR, DR, RR configurations show similar tails near $\alpha=-1$. Again, these configurations have a $50 \%$ probability of being an XD configuration (here $\mathrm{X}$ just means that the orientation of the smaller $\mathrm{BH}$ is unimportant).

In Fig. 20, we show the integrated probability $\Pi(v)$ for a recoil $v$ or larger, where

$$
\Pi(v)=\int_{v}^{\infty} P(\nu) d \nu,
$$

and $P(\nu)$ is the probability for a recoil with speed $\nu<$ $v<\nu+d \nu$.

We can thus consider a scenario where coherent accretion aligned the smaller $\mathrm{BH}$ spin but left the spin of larger $\mathrm{BH}$ either aligned or counteraligned (with equal probability). For such a UR configuration, we find the probability for $V>250 \mathrm{~km} \mathrm{~s}^{-1}$ is nearly $23 \%$, while the probability for $V>400 \mathrm{~km} \mathrm{~s}^{-1}$ is $8.4 \%$. If we assume that both BHs are equally likely to be aligned or counteraligned, the probabilities reduce to $19 \%$ and $4.2 \%$, respectively. While these recoil velocities are enough to 

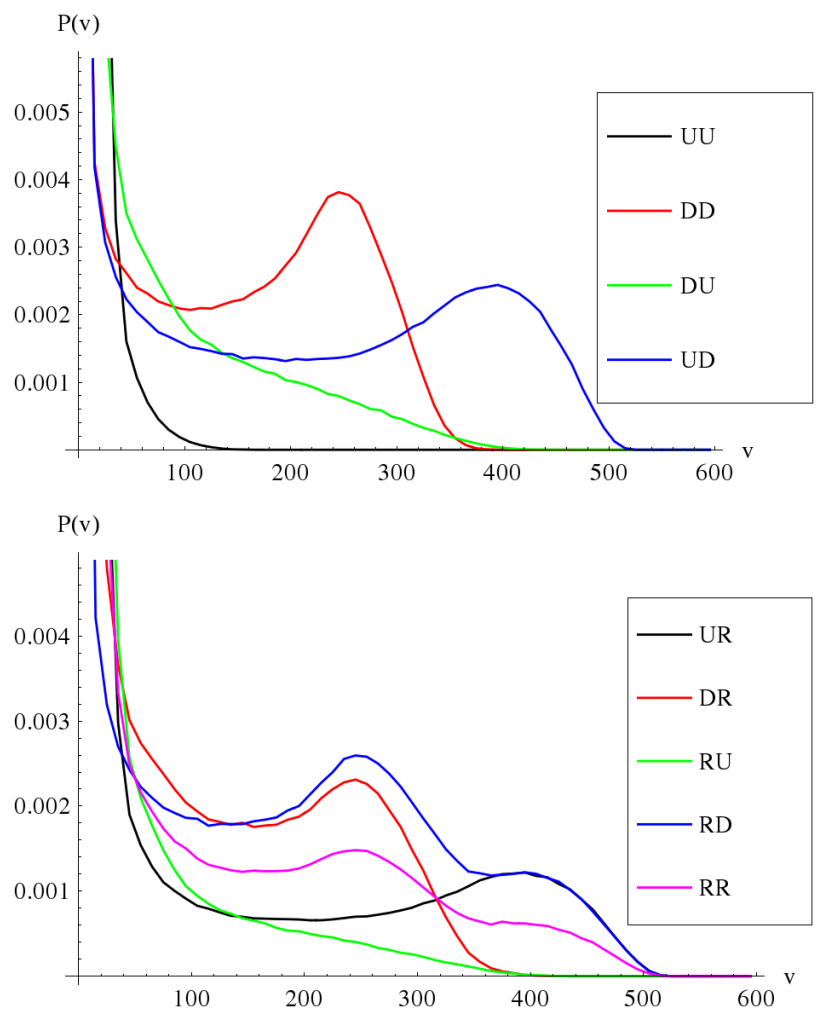

FIG. 17: The probability $P(v)$ of the remnant $\mathrm{BH}$ recoiling with speed $v$ assuming a distribution of progenitor binaries with spin-magnitude given by the cold accretion model of [7] and mass ratio distribution given by 71, and assuming the first (smaller) $\mathrm{BH}$ or second (larger) $\mathrm{BH}$ is always aligned (U), always counteraligned (D), or randomly (R) distributed with equal probability of being aligned or counteraligned.

expel the merged BHs from galaxies similar to the milky way, they are not enough for the BHs to escape from much larger galaxies. Nevertheless, these recoils can still produce observational effects such as displacement of the central $\mathrm{BH}$ from the galactic core or a disturbance in the velocity field of nearby stars 4. We also note that these recoil velocities probabilities represent a lower bound for large recoils since we assumed exact alignment (or counteralignment) of the spins with the orbital angular momentum and components of the spin on the orbital plane can lead to very large recoils 6, 8, even for relatively small misalignment angles, i.e., a few degrees.

\section{CONCLUSIONS AND DISCUSSION}

We studied the merger remnant of nonprecessing BHBs as a function of the individual $\mathrm{BH}$ spins and mass ratio. As accretion [27, 74] and resonances [28, 29] align spins with the orbital angular momentum, this represents an important subcase of the more general, 7 dimensional parameter space of binaries, that includes arbitrary orientation of the spins. The study performed here allowed us
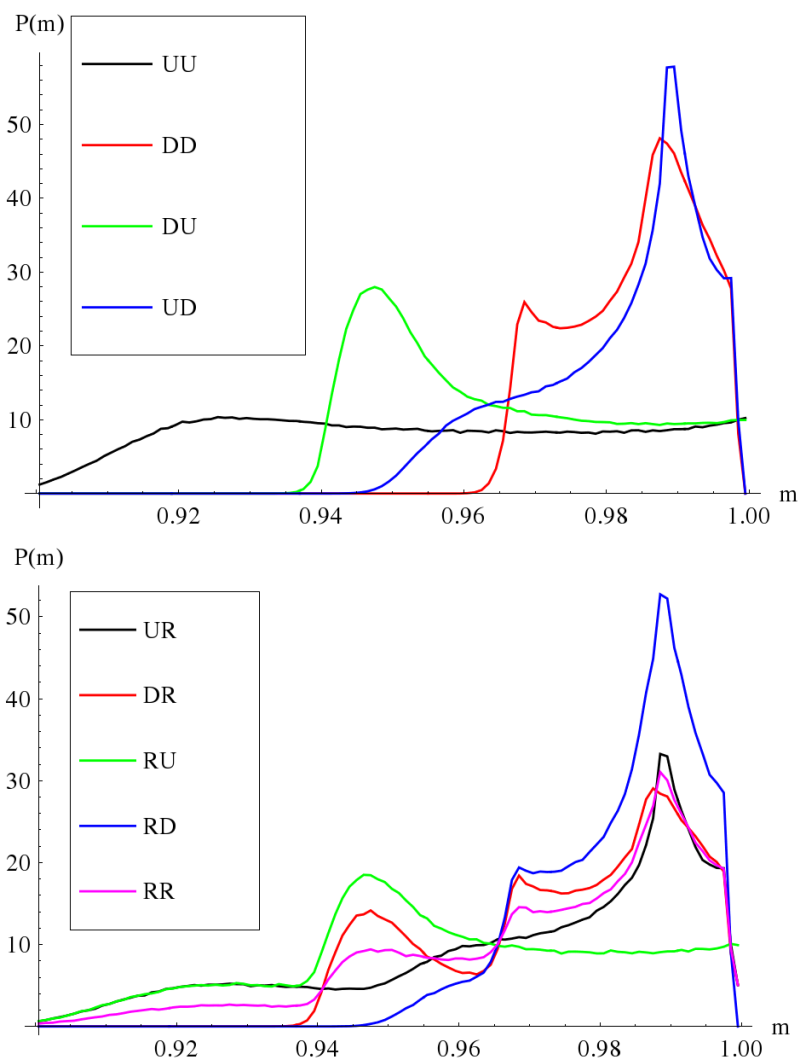

FIG. 18: The probability $P(m)$ of the remnant $\mathrm{BH}$ having mass $m$ (in units of the initial mass $M_{1}+M_{2}$ ) assuming a distribution of progenitor binaries with spin-magnitude given by the cold accretion model of [7] and mass ratio distribution given by [71] and assuming the first (smaller) $\mathrm{BH}$ or second (larger) BH is always aligned (U), always counteraligned (D), or randomly $(\mathrm{R})$ distributed with equal probability of being aligned or counteraligned.

to use the unified phenomenological description of a BHB merger developed in [8, 55, to model the recoil (17), remnant mass (14), and spin (16), with expected accuracies to within $3 \%, 1 \%$, and $1 \%$ relative errors, respectively.

We found that the spin contribution to the recoil can add to, or subtract from, the component of the recoil due to unequal masses, with (partial) cancellation occurring when the larger $\mathrm{BH}$ spin is aligned with the orbital angular momentum. On the other hand, when the larger BH spin is counteraligned, the two components of the recoil add, leading to larger recoils at intermediate mass ratios. We find that the maximum recoil occurs for $q \sim 0.62$.

Also note that the new maximum of the recoil (See Fig. 21) represents a modest increase in the maximum value itself (nearly 17\%). However, just like for the case of the hangup kicks [6], the most important effect is that the volume of parameter space leading to large recoils is much larger, i.e., the UD configurations have $\left(V_{\text {recoil }}>\right.$ $200 \mathrm{~km} \mathrm{~s}^{-1}$ ) with a $52 \%$ probability.

Another similarity with the hangup kick effect is the need to incorporate terms beyond linear in the spins (and 


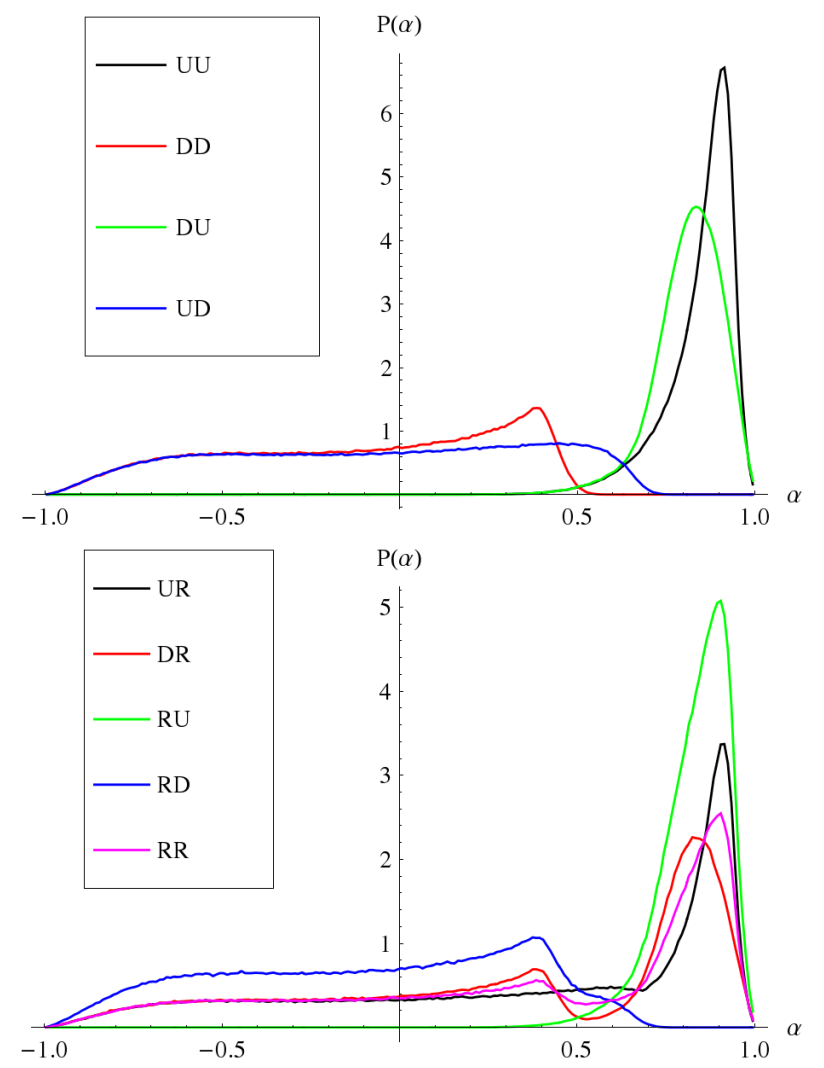

FIG. 19: The probability $P(\alpha)$ of the remnant BH having dimensionless spin $\alpha$ assuming a distribution of progenitor binaries with spin-magnitude given by the cold accretion model of [7] and mass ratio distribution given by 71 and assuming the first (smaller) BH or second (larger) BH is always aligned (U), always counteraligned (D), or randomly (R) distributed with equal probability of being aligned or counteraligned.

mass ratio) to accurately model the final recoil (and mass and spin). This underlines the inherently nonlinear nature of general relativity, in particular when modeling the highly dynamical regime of BHB mergers.

This provides the opportunity for an important test of general relativity in its strong field realm. Searches for observational effects from recoiling black holes are well underway. This includes searches for large differential red/blue shifts from AGN (see 32 for a review), and distortions in the dynamics of the core of galaxies (see [33. for the latest observation that the lack of black holes in bright cluster galaxies might be the result of large kicks).

We finally note that the use of gravitational waveforms from aligned and antialigned spins proves to be of great help for detection algorithms [75] used by laser interferometer observatories. Our models for the final mass and spin from the merger of two black holes can be used to produce more accurate semianalytic models of such waveform templates, which may also be used for parameter estimation.
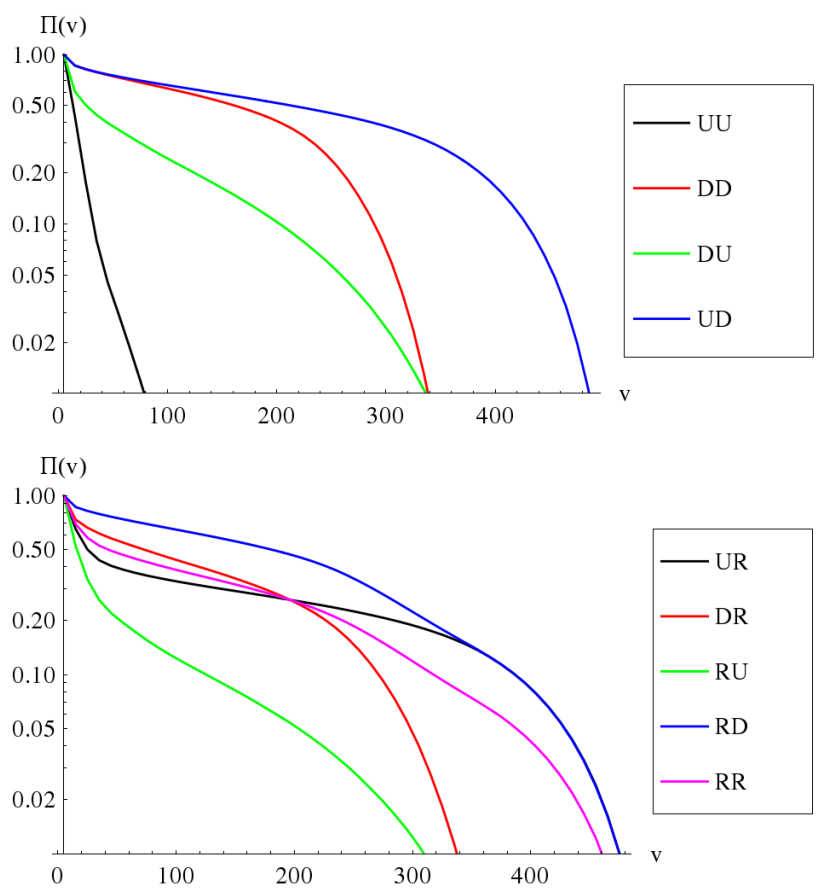

FIG. 20: The integrated probability $\Pi(v)$ of the remnant $\mathrm{BH}$ recoiling with speed $v$ or larger assuming a distribution of progenitor binaries with spin-magnitude given by the cold accretion model of [7] and mass ratio distribution given by [71] and assuming the first (smaller) $\mathrm{BH}$ or second (larger) $\mathrm{BH}$ is always aligned (U), always counteraligned (D), or randomly (R) distributed with equal probability of being aligned or counteraligned.

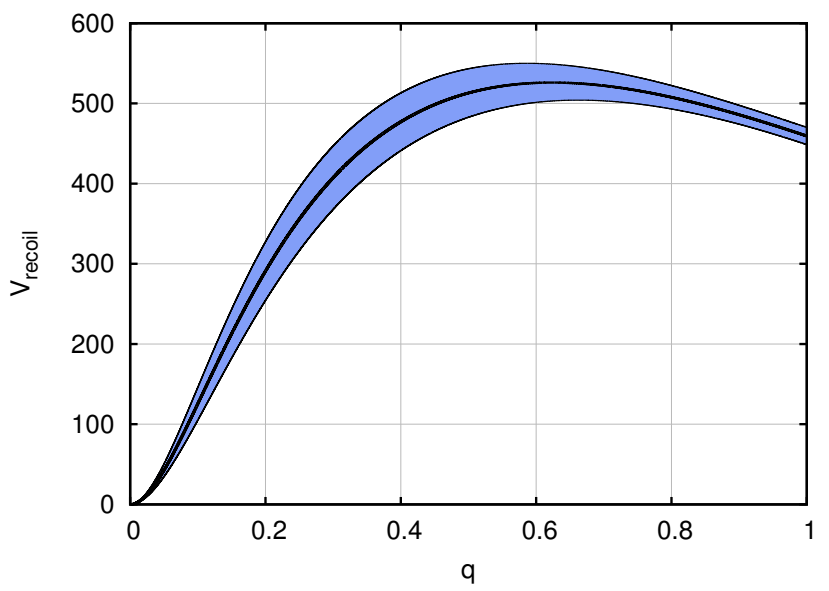

FIG. 21: The recoil velocity for the UD configuration with the small black hole spin aligned with the orbital angular momentum and the large hole spin counteraligned as a function of the mass ratio as predicted by Eq. 17. The maximum recoil of $526 \pm 23 \mathrm{~km} \mathrm{~s}^{-1}$ is reached at $q=0.6235 \pm 0.038$, with maximally spinning holes, $\alpha_{1}=1.0, \alpha_{2}=-1.0$. The shaded area represents the estimated errors. 
TABLE VII: A and B case studies with BHs at two different initial separations with two sets of parameters from estimated quasicircular orbits.

\begin{tabular}{lcccccccccccc}
\hline \hline Config. & $x_{1} / m$ & $x_{2} / m$ & $P / m$ & $m_{1}^{p} / m$ & $m_{2}^{p} / m$ & $S_{1} / m^{2}$ & $S_{2} / m^{2}$ & $m_{1}^{H} / m$ & $m_{2}^{H} / m$ & $M_{\text {ADM }} / m$ & $a_{1} / m_{1}^{H}$ & $a_{2} / m_{2}^{H}$ \\
\hline A_DU0.8 & -4.9832 & 4.5267 & 0.09905 & 0.30178 & 0.30168 & -0.2 & 0.2 & 0.5 & 0.5 & 0.98951 & -0.8 & 0.8 \\
B_DU0.8 & -4.5465 & 4.4303 & 0.10557 & 0.30377 & 0.30366 & -0.20465 & 0.20465 & 0.5053 & 0.5053 & 1 & -0.8 & 0.8 \\
\hline \hline
\end{tabular}

\section{Acknowledgments}

The authors gratefully acknowledge the NSF for financial support from Grants PHY-1305730, PHY1212426, PHY-1229173, AST-1028087, PHY-0969855, OCI-0832606, and DRL-1136221. Computational resources were provided by XSEDE allocation TGPHY060027N, and by NewHorizons and BlueSky Clusters at Rochester Institute of Technology, which were supported by NSF grant No. PHY-0722703, DMS0820923, AST-1028087, and PHY-1229173.

\section{Appendix A: Analysis of the sources of errors and robustness of remnant properties in the $\mathrm{BHB}$ simulations}

In order to assess the robustness of our results with respect to the different sources of errors and the various approximations that we use in our simulations, we study in detail an equal-mass BHB in a UD configuration (with spins $\alpha=0.8$ ) starting from two different initial separations. We vary the resolutions, grid structure, waveform extraction radii, and the number of $\ell$ modes used in the construction of the radiated linear momentum. The initial data parameters for the two configurations, denoted here by $\mathrm{A}$ and $\mathrm{B}$, are given in Table VII.

Case A represents a prototypical configuration of the runs in this paper while the case B was first studied in

Interestingly, we see that while the measured recoils from the $\mathrm{A}$ and $\mathrm{B}$ simulations differ by $\sim 30 \mathrm{~km} \mathrm{~s}^{-1}$ at lower resolution and smaller radii, they approach each other as both the resolution and extraction radii increases. The differences in the extrapolated recoil for the highest resolution $\mathrm{A}$ and $\mathrm{B}$ configuration is smaller than $10 \mathrm{~km} \mathrm{~s}^{-1}$.

In Fig. 23 we show the recoil extrapolated to infinity versus the number of $\ell$ modes. Interestingly, for the $\mathrm{B}$ configuration, using all modes up through $\ell=4$ appears to be sufficient, while for the A configuration, there is a noticeable change in the recoil when adding the $\ell=5$ modes.

Based on the results in Figs. 22 and 23, we used the medium resolution grid structure (i.e., A-M in the figures) and summed all modes up through $\ell=6$ when calculating the recoils given in the tables and figures of this
Ref. 55, where we also performed a convergence study of that configuration. In this work, we use a grid structure with between 9 and 11 levels of refinement, depending on mass ratio and spin. For all new simulations, the outer boundary was placed at $400 \mathrm{M}$ with a resolution of $4 M$ on the coarsest level and a resolution of $1 M$ in the wavezone. The finest level around each $\mathrm{BH}$ was as wide as twice the diameter of the relaxed horizon (the number of points across each horizon was between 28 and 60). In addition, for the highly-spinning horizons, we added an additional level inside the horizon of width roughly half of the horizon diameter. We also performed similar runs but with resolutions in the wavezone of $M / 0.88$ and $M / 1.2$.

Since in the current work, we use a different refinement level grid structure than in Ref. [55], we also perform a new set of convergence simulations for case B using the newer grid structures.

Aside from truncation errors due to finite resolution, the simulation results will depend on the extraction radii. Hence we also consider different extraction radii and extrapolations to null infinity. While the location of the observers in the set of runs in Ref. [55] was restricted to the $R_{o b s} / m=60-100$ range in the runs of this paper we extended this to $R_{o b s} / m=190$ and, in addition, locate the extraction radii equidistant in $1 / R$, with $R_{\text {obs }} / \mathrm{m}=$ 75, 80.4, 86.7, 94.0, 102.6, 113.0, 125.7, 141.7, 162.3, 190.0. The results of such studies is displayed in Fig. 22

work. We also note that, because the recoils from the A and B configurations did approach each other, effects due to finite starting separation are reasonably mitigated for initial separations of between $9 M$ and $10 M$ and above.

In conclusion we see that in order to have a robust measure of the recoil we need to consider BHBs with sufficiently large initial separations, medium resolution, and we need to sum over modes up through $\ell=6$.

In Fig. 24, we plot the radiated energy and the ratio of the final to initial mass derived from the radiated energy as a function of $1 / r$ and $\ell_{\max }$. We see that $\mathrm{A}$ and $\mathrm{B}$ configuration approach each other with an increase in resolution and larger observer radii. We also note that there are only small errors introduced by using $\ell_{\max }$ as small as $\ell=4$. For the radiated angular momentum (see Fig. 25), the extrapolation error dominates, and while the $\mathrm{A}$ and $\mathrm{B}$ configurations results seems to converge to 


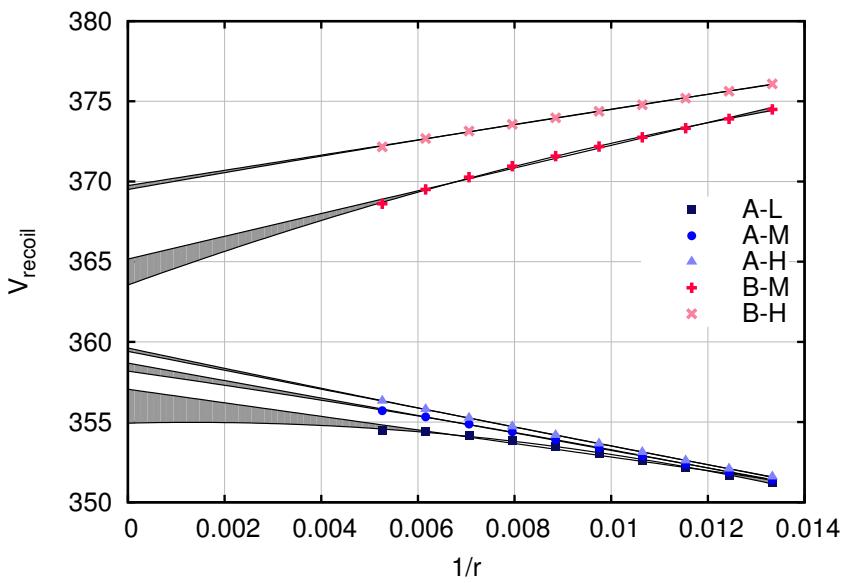

FIG. 22: The recoil velocity as computed at a given extraction radius: $75 M-190 M$ and extrapolations to infinity. The different curves correspond to the two initial separations labeled as A and B and as a function of resolution (Low - Medium - High) refined by a global factor 1.2. The shaded regions are those points contained between a linear and quadratic extrapolation of the data (least squares fit).

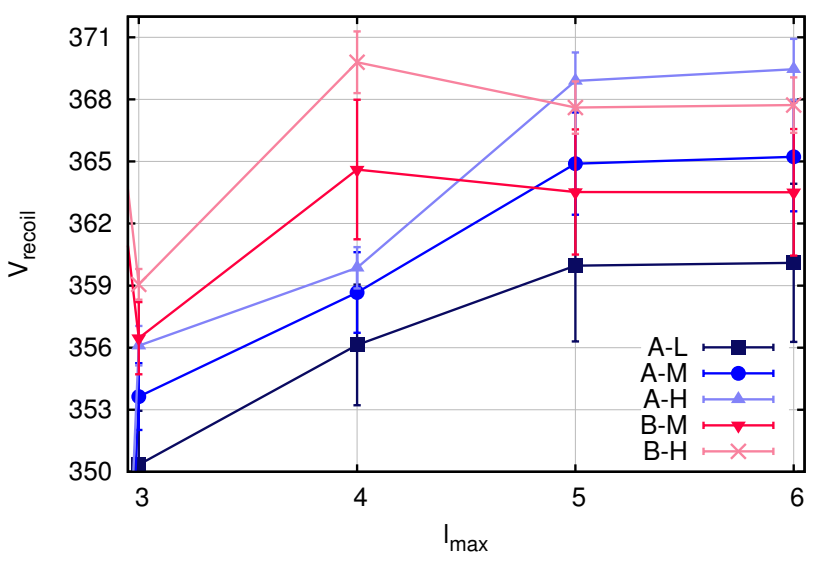

FIG. 23: The dependence of the computed recoil velocity on the number of $\ell$ modes used to construct the radiated linear momentum. Here all modes with $\ell \leq \ell_{\max }$ were used and we show the recoil for the $\mathrm{A}$ and $\mathrm{B}$ configurations for the Low, Medium, and High resolution runs.

each other, the error bars are quite large. Again, we see that summing up through $\ell=4$ is sufficient to obtain the final remnant spin.

One final note, for the runs presented in the main body of the paper, we use extraction radii up to $r=102.6 \mathrm{~m}$, rather than $r=190 \mathrm{~m}$. We did this because we observed, at our working resolutions, a dissipation effect at larger radii in which the amplitude of $r \psi_{4}$ steadily decreases with radius in the outer zone.

We apply all these criteria to the rest of the new simulations we perform in this paper to ensure similar error bars as the ones presented in this Appendix. Note also that a similar, but independent, waveform-error analysis
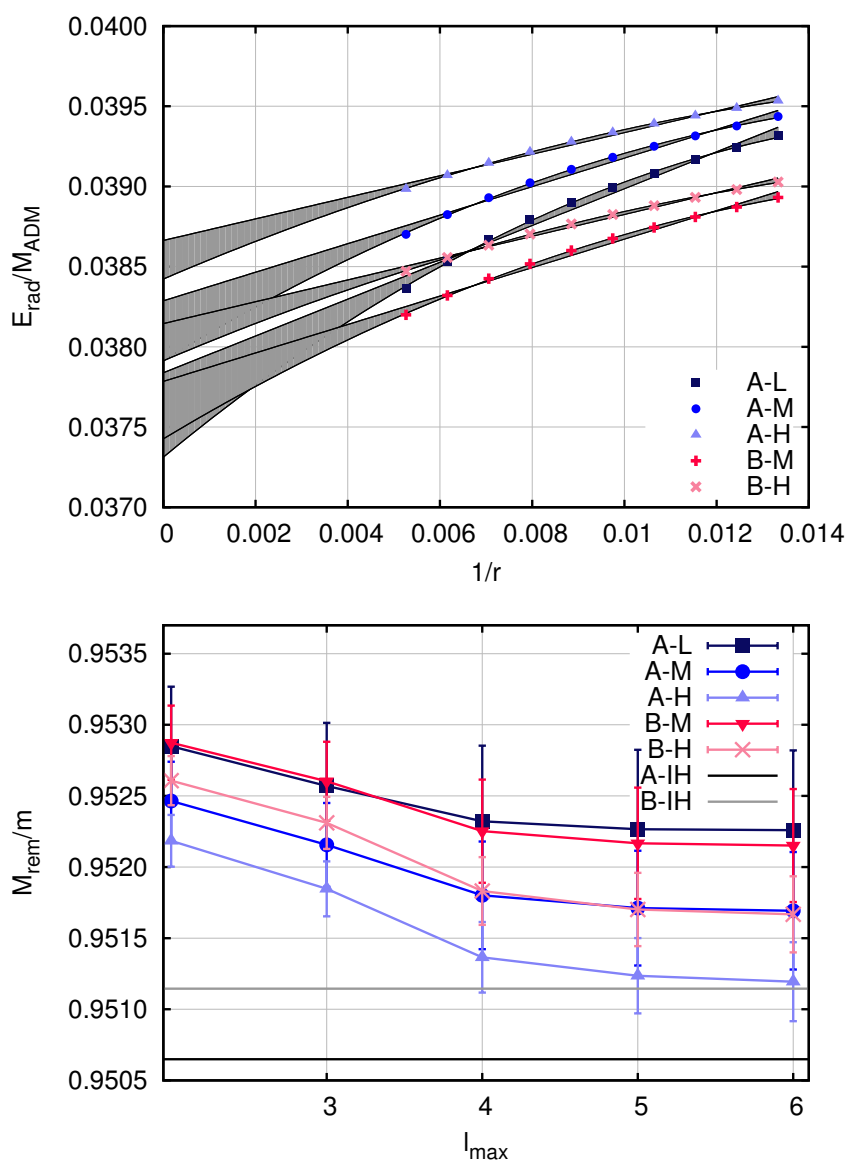

FIG. 24: Above: The radiated energy as computed at a given extraction radius: $75 M-190 M$ and extrapolations to infinity. The different curves correspond to the two initial separations labeled as A and B and as a function of resolution (Low - Medium - High) refined by a global factor 1.2 . The shaded regions are those points contained between a linear and quadratic extrapolation of the data (least squares fit). Below: The dependence of the computed radiated energy on the number of $\ell$ modes used to construct it. Here all modes with $\ell \leq \ell_{\max }$ were used. The black and gray lines labeled with "IH" are the associated final mass calculated from the $\mathrm{BH}$ horizon. On this scale, all resolutions are on top of one another, so only one line is shown.

was carried out in Ref. [76].
[1] F. Pretorius, Phys. Rev. Lett. 95, 121101 (2005), grqc/0507014.
[2] M. Campanelli, C. O. Lousto, P. Marronetti, and Y. Zlochower, Phys. Rev. Lett. 96, 111101 (2006), gr- 

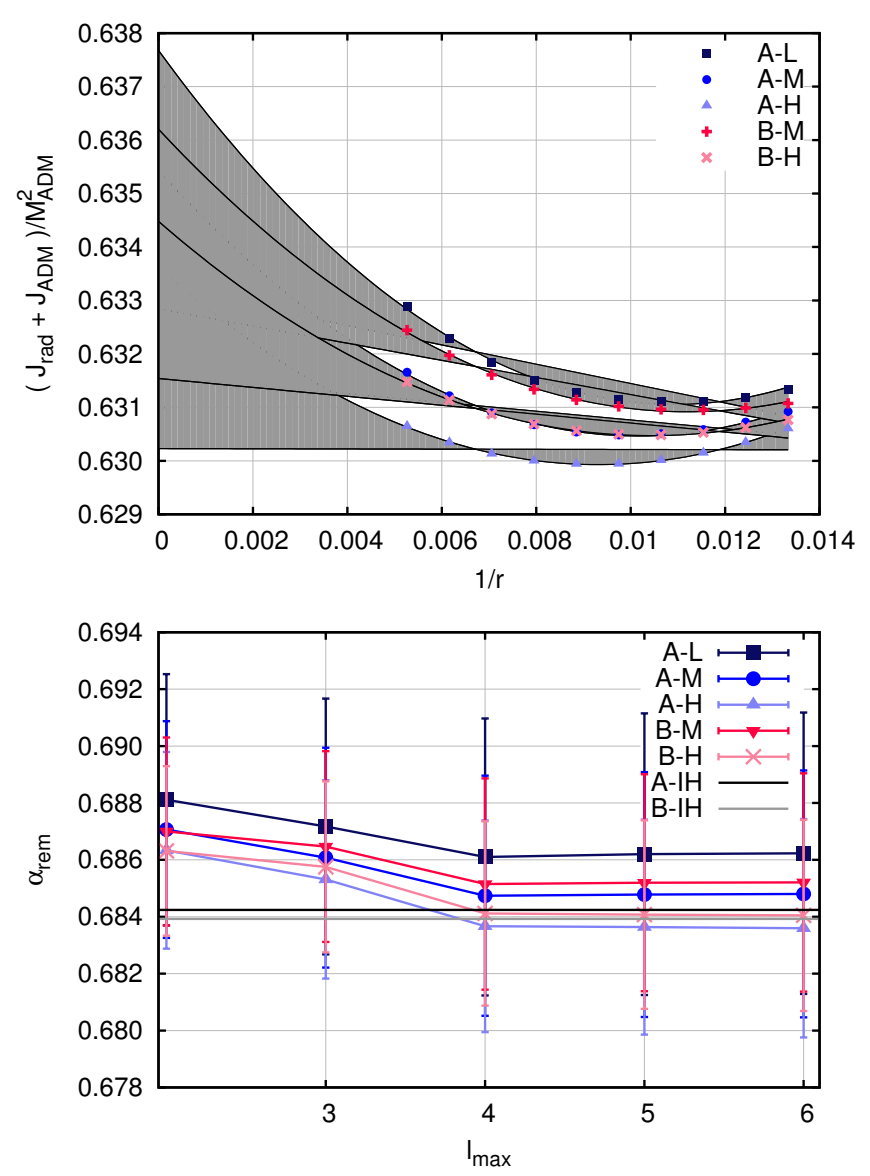

FIG. 25: Above: The radiated angular momentum as computed at a given extraction radius: $75 M-190 M$ and extrapolations to infinity. The different curves correspond to the two initial separations labeled as A and B and as a function of resolution (Low - Medium - High) refined by a global factor 1.2. The shaded regions are those points contained between a linear and quadratic extrapolation of the data (least squares fit). Below: The dependence of the computed radiated angular momentum on the number of $\ell$ modes used to construct it. Here all modes with $\ell \leq \ell_{\max }$ were used. The black and gray lines labeled with "IH" are the associated final spin calculated from the $\mathrm{BH}$ horizon. On this scale, all resolutions are on top of one another, so only one line is shown.

qc/0511048.

[3] J. G. Baker, J. Centrella, D.-I. Choi, M. Koppitz, and J. van Meter, Phys. Rev. Lett. 96, 111102 (2006), grqc/0511103.

[4] M. Campanelli, C. O. Lousto, Y. Zlochower, and D. Merritt, Astrophys. J. 659, L5 (2007), gr-qc/0701164.

[5] J. A. González, M. D. Hannam, U. Sperhake, B. Brugmann, and S. Husa, Phys. Rev. Lett. 98, 231101 (2007), gr-qc/0702052.

[6] C. O. Lousto and Y. Zlochower, Phys. Rev. Lett. 107, 231102 (2011), 1108.2009

[7] C. O. Lousto, Y. Zlochower, M. Dotti, and M. Volonteri, Phys. Rev. D85, 084015 (2012), 1201.1923.

[8] C. O. Lousto and Y. Zlochower, Phys. Rev. D87, 084027 (2013), 1211.7099 .
[9] C. O. Lousto and Y. Zlochower, Phys. Rev. D83, 024003 (2011), 1011.0593.

[10] Y. Zlochower, M. Campanelli, and C. O. Lousto, Class. Quant. Grav. 28, 114015 (2011), 1011.2210.

[11] C. O. Lousto, M. Campanelli, Y. Zlochower, and H. Nakano, Class. Quant. Grav. 27, 114006 (2010), 0904.3541.

[12] C. O. Lousto and Y. Zlochower, Phys. Rev. D79, 064018 (2009), 0805.0159.

[13] S. C. Noble, B. C. Mundim, H. Nakano, J. H. Krolik, M. Campanelli, Y. Zlochower, and N. Yunes, Astrophys. J. 755, 51 (2012), 1204.1073.

[14] T. Bode, T. Bogdanovic, R. Haas, J. Healy, P. Laguna, et al., Astrophys. J. 744, 45 (2012), 1101.4684.

[15] T. Bogdanovic, T. Bode, R. Haas, P. Laguna, and D. Shoemaker, Class. Quant. Grav. 28, 094020 (2011), 1010.2496.

[16] T. Bode, R. Haas, T. Bogdanovic, P. Laguna, and D. Shoemaker, Astrophys. J. 715, 1117 (2010), 0912.0087

[17] R. Gold, V. Paschalidis, Z. B. Etienne, S. L. Shapiro, and H. P. Pfeiffer (2013), 1312.0600.

[18] S. L. Shapiro, Phys. Rev. D87, 103009 (2013), 1304.6090.

[19] B. D. Farris, R. Gold, V. Paschalidis, Z. B. Etienne, and S. L. Shapiro, Phys. Rev. Lett. 109, 221102 (2012), 1207.3354.

[20] B. D. Farris, Y. T. Liu, and S. L. Shapiro, Phys. Rev. D84, 024024 (2011), 1105.2821.

[21] C. Palenzuela, L. Lehner, and S. L. Liebling, Science 329, 927 (2010), 1005.1067.

[22] C. Palenzuela, L. Lehner, and S. Yoshida, Phys. Rev. D81, 084007 (2010), 0911.3889.

[23] M. Megevand, M. Anderson, J. Frank, E. W. Hirschmann, L. Lehner, et al., Phys. Rev. D80, 024012 (2009), 0905.3390.

[24] C. Palenzuela, M. Anderson, L. Lehner, S. L. Liebling, and D. Neilsen, Phys. Rev. Lett. 103, 081101 (2009), 0905.1121.

[25] T. Bogdanovic, C. S. Reynolds, and M. C. Miller, Astrophys. J. 661, L147 (2007), astro-ph/0703054.

[26] M. Dotti, M. Volonteri, A. Perego, M. Colpi, M. Ruszkowski, and F. Haardt, mnras 402, 682 (2010), 0910.5729 .

[27] M. Coleman Miller and J. H. Krolik, Astrophys. J. 774, 43 (2013), 1307.6569.

[28] J. D. Schnittman, Phys. Rev. D70, 124020 (2004), astro$\mathrm{ph} / 0409174$.

[29] D. Gerosa, M. Kesden, E. Berti, R. O'Shaughnessy, and U. Sperhake, Phys. Rev. D87, 104028 (2013), 1302.4442.

[30] M. Dotti, M. Colpi, S. Pallini, A. Perego, and M. Volonteri, Astrophys. J. 762, 68 (2013), 1211.4871.

[31] A. Sesana, E. Barausse, M. Dotti, and E. M. Rossi (2014), 1402.7088 .

[32] S. Komossa, Adv. Astron. 2012, 364973 (2012), 1202.1977.

[33] D. Gerosa and A. Sesana (2014), 1405.2072.

[34] J. A. González, U. Sperhake, B. Brugmann, M. Hannam, and S. Husa, Phys. Rev. Lett. 98, 091101 (2007), grqc/0610154.

[35] F. Herrmann, I. Hinder, D. Shoemaker, P. Laguna, and R. A. Matzner, Astrophys. J. 661, 430 (2007), grqc/0701143.

[36] M. Koppitz, D. Pollney, C. Reisswig, L. Rezzolla, J. Thornburg, et al., Phys. Rev. Lett. 99, 041102 (2007), 
gr-qc/0701163.

[37] J. G. Baker et al., Astrophys. J. 682, L29 (2008), 0802.0416 .

[38] C. O. Lousto and Y. Zlochower, Phys. Rev. D77, 044028 (2008), 0708.4048.

[39] L. E. Kidder, Phys. Rev. D52, 821 (1995), grqc/9506022.

[40] E. Racine, A. Buonanno, and L. E. Kidder, Phys. Rev. D80, 044010 (2009), 0812.4413.

[41] M. Campanelli, C. O. Lousto, and Y. Zlochower, Phys. Rev. D74, 041501(R) (2006), gr-qc/0604012.

[42] M. Ansorg, B. Brügmann, and W. Tichy, Phys. Rev. D70, 064011 (2004), gr-qc/0404056.

[43] S. Brandt and B. Brügmann, Phys. Rev. Lett. 78, 3606 (1997), gr-qc/9703066.

[44] Y. Zlochower, J. G. Baker, M. Campanelli, and C. O. Lousto, Phys. Rev. D72, 024021 (2005), gr-qc/0505055.

[45] P. Marronetti, W. Tichy, B. Brügmann, J. Gonzalez, and U. Sperhake, Phys. Rev. D77, 064010 (2008), 0709.2160.

[46] C. O. Lousto and Y. Zlochower, Phys. Rev. D77, 024034 (2008), 0711.1165.

[47] Cactus Computational Toolkit home page: http: //cactuscode.org.

[48] Einstein Toolkit home page: http://einsteintoolkit.org.

[49] E. Schnetter, S. H. Hawley, and I. Hawke, Class. Quant. Grav. 21, 1465 (2004), gr-qc/0310042.

[50] J. Thornburg, Class. Quant. Grav. 21, 743 (2004), grqc/0306056.

[51] O. Dreyer, B. Krishnan, D. Shoemaker, and E. Schnetter, Phys. Rev. D67, 024018 (2003), gr-qc/0206008.

[52] M. Campanelli and C. O. Lousto, Phys. Rev. D59, 124022 (1999), gr-qc/9811019.

[53] J. Winicour, in General Relativity and Gravitation Vol 2, edited by A. Held (Plenum, New York, 1980), pp. 71-96.

[54] C. O. Lousto and Y. Zlochower, Phys. Rev. D76, 041502(R) (2007), gr-qc/0703061.

[55] C. O. Lousto and Y. Zlochower (2013), 1312.5775.

[56] A. Ori and K. S. Thorne, Phys. Rev. D 62, 124022 (2000).

[57] H. Nakano, M. Campanelli, C. O. Lousto, and Y. Zlochower, Class. Quant. Grav. 28, 134005 (2011),
1011.2767 .

[58] C. M. Hirata, Phys.Rev. D83, 104024 (2011), 1011.4987.

[59] M. van de Meent (2014), 1406.2594.

[60] http://www.black-holes.org/waveforms

[61] D. Pollney et al., Phys. Rev. D76, 124002 (2007), 0707.2559.

[62] T. Damour, Phys. Rev. D64, 124013 (2001), grqc/0103018.

[63] C. O. Lousto, H. Nakano, Y. Zlochower, and M. Campanelli, Phys. Rev. D82, 104057 (2010), 1008.4360.

[64] H. Nakano, Y. Zlochower, C. O. Lousto, and M. Campanelli, Phys. Rev. D84, 124006 (2011), 1108.4421.

[65] E. Barausse and L. Rezzolla, Astrophys. J. Lett. 704, L40 (2009), 0904.2577.

[66] E. Barausse, V. Morozova, and L. Rezzolla, Astrophys. J. 758, 63 (2012), 1206.3803.

[67] L. Rezzolla, R. P. Macedo, and J. L. Jaramillo, Phys. Rev. Lett. 104, 221101 (2010), 1003.0873.

[68] M. Campanelli, C. O. Lousto, Y. Zlochower, and D. Merritt, Phys. Rev. Lett. 98, 231102 (2007), gr-qc/0702133.

[69] L. Rezzolla et al., Astrophys. J. 674, L29 (2008), arXiv:0710.3345 [gr-qc].

[70] D. A. Hemberger, G. Lovelace, T. J. Loredo, L. E. Kidder, M. A. Scheel, et al., Phys. Rev. D88, 064014 (2013), 1305.5991.

[71] Q. Yu, Y. Lu, R. Mohayaee, and J. Colin, Astrophys. J. 738, 92 (2011), 1105.1963.

[72] K. R. Stewart, J. S. Bullock, E. J. Barton, and R. H. Wechsler, Astrophys. J. 702, 1005 (2009), 0811.1218.

[73] P. F. Hopkins, K. Bundy, D. Croton, L. Hernquist, D. Keres, et al., Astrophys. J. 715, 202 (2010), 0906.5357.

[74] K. A. Sorathia, J. H. Krolik, and J. F. Hawley, Astrophys. J. 777, 21 (2013), 1309.0290.

[75] S. Privitera, S. R. P. Mohapatra, P. Ajith, K. Cannon, N. Fotopoulos, et al., Phys. Rev. D89, 024003 (2014), 1310.5633

[76] I. Hinder et al. (Perimeter Institute for Theoretical Physics), Class. Quant. Grav. 31, 025012 (2014), 1307.5307. 